\title{
Modelling the Self-Assembly of Silica-Based Mesoporous Materials
}

\author{
Miguel Jorge ${ }^{1 *}$, Andrew W. Milne ${ }^{1}$, Olivia N. Sobek ${ }^{1}$, Alessia Centi ${ }^{1,}$, \\ Germán Pérez-Sánchez², José R. B. Gomes²
}

1) Department of Chemical and Process Engineering, University of Strathclyde, 75 Montrose Street, Glasgow G1 1XJ, United Kingdom

2) CICECO - Aveiro Institute of Materials, Department of Chemistry, University of Aveiro, Campus Universitário de Santiago, 3810-193 Aveiro, Portugal

\author{
*corresponding author email: miguel.jorge@strath.ac.uk \\ $\S$ Present address: Max Planck Institute for Polymer Research, Ackermannweg 10, D- \\ 55128 Mainz, Germany
}

\begin{abstract}
Periodic Mesoporous Silicas (PMS) are one of the prime examples of templated porous materials - there is a clear connection between the porous network structure and the supramolecular assemblies formed by surfactant templates. This opens the door for a high degree of control over the material properties by tuning the synthesis conditions, and has led to their application in a wide range of fields, from gas separation and catalysis to drug delivery. However, such control has not yet come to full fruition, largely because a detailed understanding of the synthesis mechanism of these materials remains elusive. In this context, molecular modelling studies of the self-assembly of silica/surfactant mesophases have arisen at the turn of the century. In this paper, we present a comprehensive review of simulation studies devoted to the synthesis of PMS materials and their hybrid organic-inorganic counterparts. As those studies span a wide range of time and length scales, a holistic view of the field affords some interesting new insight into the synthesis mechanisms. We expect simulation studies of this complex but fascinating topic to increase significantly as computer architectures become increasingly powerful, and we present our view to the future of this field of research.
\end{abstract}

Keywords: MCM-41, SBA-15, Organosilica, Bio-inspired, Coarse-grained 


\section{Introduction}

In its broadest sense, Periodic Mesoporous Silicas (PMS) are materials composed of an amorphous silicon dioxide $\left(\mathrm{SiO}_{2}\right)$ scaffold that forms a porous structure with mesoscale ordering. The ordering of the pores is brought about by the use of supramolecular templates, usually molecular surfactants or block co-polymers, which self-assemble in an aqueous solution containing a silica source. Depending on the conditions of the synthesis and nature of the surfactant, different mesostructures will form in solution, leading to porous materials with potentially controllable structural features. PMS are therefore a flagship success case of the templated synthesis approach for porous material production [1]. Their discovery has been almost exclusively attributed to the work of Mobil scientists in 1992 [2, 3]. In fact, the archetypal PMS material, and arguably the most studied member of this family, is MCM-41 (MCM stands for Mobil Composition of Matter), which exhibits roughly cylindrical pores organised in a hexagonal array. However, Di Renzo et al. [4] have shown that a recipe published in a 1971 patent by Chiola et al. [5] leads to the synthesis of a PMS material that is very similar to MCM-41. Nevertheless, Beck et al. [2] were undoubtedly the first to recognise the importance of their discovery, and their work generated great interest in the material science community. Their papers sparked a great number of subsequent studies reporting new mesoporous materials obtained through similar template-based routes, in a phenomenon perhaps not very different from the much more recent hype surrounding Metal-Organic Frameworks [6]. Some of the most relevant advances in the early years following the paper by Beck et al. were the extension of PMS synthesis to a wide range of $\mathrm{pH}$ conditions [7], the discovery of block copolymer templated materials, of which the most well-known example is SBA-15 [8], the synthesis of bio-inspired mesoporous silica through the use of amine-based surfactants [9, 10], and the 
incorporation of organic groups on the pore walls of the material, leading to the discovery of Periodic Mesoporous Organosilicas (PMO) [11-13]. PMS materials and their derivatives are now applied in a variety of fields, including gas separation and storage, catalysis, drug delivery, sensors, energy storage, and imaging [14, 15].

This surge in the discovery of new mesoporous silica materials was closely followed by attempts to better understand their synthesis process, mainly through experimental means. Despite several notable advances, however, a complete understanding of the synthesis process of even the simplest of these materials, MCM41, has still not been achieved. The main challenges lie in the complex nature of the process, involving phenomena like self-assembly, chemical reactions, nucleation, and phase separation, all taking place simultaneously within the precursor solution, as well as in the wide range of time and length scales that are relevant to the material formation [16]. This makes it very difficult to probe the entire synthesis mechanism in situ using a single technique, or even a combination of experimental techniques under the same conditions. The nature of the synthesis process, described above, also poses formidable challenges to theoreticians, and it is perhaps not surprising that it was not until 2001 that the first attempts to describe the formation of PMS materials through computer simulation were published [17]. In fact, it is fair to say that our theoretical understanding of the PMS synthesis process has significantly lagged behind (arguably by about two decades) experimental studies into this process. However, in recent years, due partly to the availability of increasingly powerful computational resources and to the development of new models and simulation algorithms, there have been significant new developments that have placed us on the verge of a complete description of the synthesis mechanism of MCM-41, and have led to new insight into the synthesis of related materials. The goal of the present paper is to present a thorough review of 
simulation studies of mesoporous silica self-assembly from solution. Although it is not our aim to review experimental studies of the PMS synthesis process (several reviews already exist on this topic $[14,18,19])$, we will make reference to key experimental papers throughout the discussion. Our paper is complementary, but distinct, from a review published recently by Auerbach et al. [16] - the latter considered both all-silica zeolites and MCM-41, while we focus on all classes of mesoporous silica and organosilica (including, but not limited to, MCM-41). Furthermore, our paper emphasises modelling approaches based on a statistical-mechanical description of the self-assembly process, and thus only those quantum mechanical studies that directly support such modelling approaches are discussed.

This paper is organised as follows. We begin by discussing the state-of-the-art regarding the synthesis of MCM-41, as this was by far the most widely studied member of the PMS family, as well as related materials also synthesised using quaternary ammonium surfactants as templates, such as MCM-48 (section 2). We then cover recent advances in modelling other silica-based materials, such as SBA-15 and bio-inspired silica (section 3), moving on to discuss simulation studies of hybrid PMO materials (section 4). We end the paper with some overall conclusions and an outlook to the future of this area of research.

\section{Synthesis of MCM-41 and related materials}

In the synthesis of PMS materials there are at least three key components - a solvent, a surfactant and a silica source - and the process is composed of at least three stages: mixing of the reagents, hydrothermal reaction with phase separation of the solid product, and removal of the template, usually through calcination. The solvent is usually 
water or a mixture of water and ethanol. In the original MCM papers $[2,3]$, the surfactants were quaternary alkylammonium salts (most prominently, cetyltrimethylammonium bromide, or CTAB), which are composed of a cationic head and a variable-length hydrophobic tail. Anionic surfactants were also used in later studies [7], but we are not aware of any attempts to date to model the synthesis of anionic mesoporous silica. The silica source is usually monosilicic acid $\left(\mathrm{Si}(\mathrm{OH})_{4}\right)$, however, since it is unstable in water, it is usually stored as tetraethylorthosilicate (TEOS - $\left.\mathrm{Si}\left(\mathrm{OCH}_{2} \mathrm{CH}_{3}\right)_{4}\right)$. When mixed with water, TEOS undergoes hydrolysis according to:

$$
\mathrm{Si}\left(\mathrm{OC}_{2} \mathrm{H}_{5}\right)_{4}+4\left(\mathrm{H}_{2} \mathrm{O}\right) \rightarrow \mathrm{Si}(\mathrm{OH})_{4}+4\left(\mathrm{C}_{2} \mathrm{H}_{5} \mathrm{OH}\right)
$$

This reaction liberates ethanol, and therefore may change the properties of the solvent. Because the hydrolysis reaction can be acid- or base-catalysed, it is very fast at both high and low pH values [20]. Under more neutral pH, however, several TEOS "arms" may remain unhydrolysed long after the silica has started to polymerise [20]. Silica polymerises according to the following polycondensation reaction:

$$
\mathrm{R}_{3} \mathrm{Si}-\mathrm{OH}+\mathrm{HO}-\mathrm{SiR}_{3} \rightarrow \mathrm{Si}_{2} \mathrm{R}_{6} \mathrm{O}+\mathrm{H}_{2} \mathrm{O}
$$

Where R is any functional group. Reaction (2) is also catalysed by both acid and base, but the nature of the condensation product, even in a simple aqueous solution, depends strongly on the synthesis $\mathrm{pH}$ - acid media tend to favour formation of cross-linked gels, while high $\mathrm{pH}$ favours formation of small compact oligomers and nanoparticles [20].

If the chemistry of silica on its own is already quite complex, with the final product relying on a delicate balance between the two reactions mentioned above, aggregation of silica particles and phase separation of the solid material, and depending strongly on solution $\mathrm{pH}$, concentration and temperature, in the presence of template 
molecules the complexity is multiplied. In the case of MCM-41, Beck et al. [2] observed that the porous structure of the final material closely resembled mesostructures formed in aqueous solutions of the template surfactant (e.g., hexagonal MCM-41, cubic MCM-48, lamellar MCM-50), and were able to show that increasing the surfactant tail length led to a relatively predictable increase in the pore diameter of hexagonal MCM41 materials. This led them to propose a liquid-crystal templating (LCT) mechanism, whereby silica condenses around pre-formed surfactant mesostructures to produce a rigid mesoporous scaffold. However, they also observed that several of those mesostructures were formed at low surfactant concentrations, conditions under which an aqueous surfactant solution would only form dispersed micelles. They therefore suggested the possibility of a second mechanism, termed cooperative templating mechanism $(\mathrm{CTM})$, whereby it is the synergistic interaction between silicates and surfactants that promotes the self-assembly of complex silica/surfactant mesostructures. The two mechanisms represent two fundamentally different views of the synthesis process - structure directing by the surfactant phase, with silica playing only a minor role, versus templating in which silica plays a major role in directing the final structure - but at the time, there was insufficient evidence to decouple the two mechanisms.

A series of experimental studies followed [21-34], using a multitude of characterisation techniques including X-ray diffraction, transmission electron microscopy, infrared and Raman spectroscopy, solid-state and solution NMR, temperature programmed desorption, gas adsorption, polarized optical microscopy, and fluorescence quenching. Although these studies undoubtedly shed new light on the PMS formation, several aspects of the synthesis mechanism remained controversial. For example, although mounting evidence in favour of the CTM has been amassed over the years [21-26], it is still unclear if the LCT is a viable mechanism under certain 
conditions [27]. Within the framework of the CTM, some researchers have argued that silicates promote a transition in the shape of surfactant micelles from small spheres to rods and even wormlike micelles at very early stages of the synthesis process [23-25], while others observed no evidence of such a transition [28, 29]. It is also still not completely clear if small silicates prefer to interact with surfactant micelles, adsorbing at their surface $[23,30,31]$, or with loose surfactant monomers $[7,28,29]$ in the early stages of the synthesis. At later stages, uncertainty remains about the way in which silica/surfactant micelles aggregate to form the liquid-crystalline mesophase, with some researchers inferring aggregation of cylindrical micelles [21, 32] and others proposing aggregation of spherical micelles followed by shape transformation within the micellar aggregate $[30,33,34]$. All these questions have profound implications on the synthesis mechanism, and on our ability to control the properties of the resulting material by tailored design.

It was in this context of uncertainty that modelling efforts to describe the PMS synthesis process were proposed as a valuable complement to experimental studies. In the following subsections, we review the state-of-the-art regarding simulations of the synthesis mechanism of MCM-41 and related materials. It is important to note that a large number of modelling studies of PMS materials have focused on building more or less realistic molecular models of the pore structure, for example by applying stochastic methods [35-37] or by modifying existing silica structures [38], in order to study their performance in gas adsorption. Those studies were mainly concerned with relating the properties of the material (e.g., pore size, wall structure) with its performance in particular applications. Instead, we are concerned here with studies that attempt to elucidate the mechanism by which these materials self-assemble from an initially disordered solution - i.e., they try to provide relationships between synthesis conditions 
and material properties. We organise this section in three more or less parallel trends; i) attempts to describe the phase equilibrium diagrams of MCM-41 precursor solutions, mostly based on lattice models; ii) attempts to describe the self-assembly mechanism of silica-surfactant mesostructures, mostly based on off-lattice models; iii) attempts to describe the silica condensation reactions in precursor solutions, mostly based on reactive potentials and/or advanced Monte Carlo algorithms.

\subsection{Phase Diagrams - Lattice Models}

Possibly the first attempt to model at least part of the self-assembly pathway with specific reference to MCM-41-type materials was the work of Siperstein and Gubbins [17, 39]. Their focus was not on obtaining atomically detailed models of MCM-41 adsorbents, but rather on exploring the phase diagrams of the ternary surfactant-solvent-silica systems. They achieved this using lattice Monte Carlo (MC) simulations in the canonical (constant $N V T$ ) ensemble, based on an earlier model of Larson et al. for surfactant self-assembly [40]. Their surfactants were comprised of head and tail beads $\left(\mathrm{H}_{\mathrm{i}} \mathrm{T}_{\mathrm{j}}\right)$; three variations thereof were investigated $-\mathrm{H}_{4} \mathrm{~T}_{4}, \mathrm{H}_{4} \mathrm{~T}_{6}$ and $\mathrm{H}_{2} \mathrm{~T}_{6}$. Each H or T bead occupied one lattice site, as did each solvent (S) or silica (I) bead. The model allowed for reptation, chain regrowth and cluster MC moves on a simple cubic lattice. Silica beads interacted strongly with the surfactant heads, while tails interacted strongly with each other to describe hydrophobic association. Silica-solvent interactions were varied, in order to describe different regimes of association.

Siperstein and Gubbins $[17,39]$ dealt initially with the binary phase diagrams; these were surfactant/solvent and surfactant/silica systems. They showed for the surfactant/solvent system that they were able to reproduce the results of Larson's model 
[40], whereby the surfactant transitions from a hexagonal to a lamellar structure by increasing the surfactant volume fraction, with a small gyroid phase region existing between the two. For the surfactant/silica system, they observed a similar trend: micelles were present at the lowest surfactant volume fraction, progressing through a hexagonal phase to perforated lamellae at the highest surfactant volume fraction (Fig. 1a). They also compared the size of the aggregates in both phases, whereby the structures in the surfactant/solvent system incorporated three times the surfactants as those in the surfactant/silica system. This is because silica-head (I-H) interactions were stronger than solvent-head $(\mathrm{S}-\mathrm{H})$ interactions in their model, leading to less phase separation.

a)

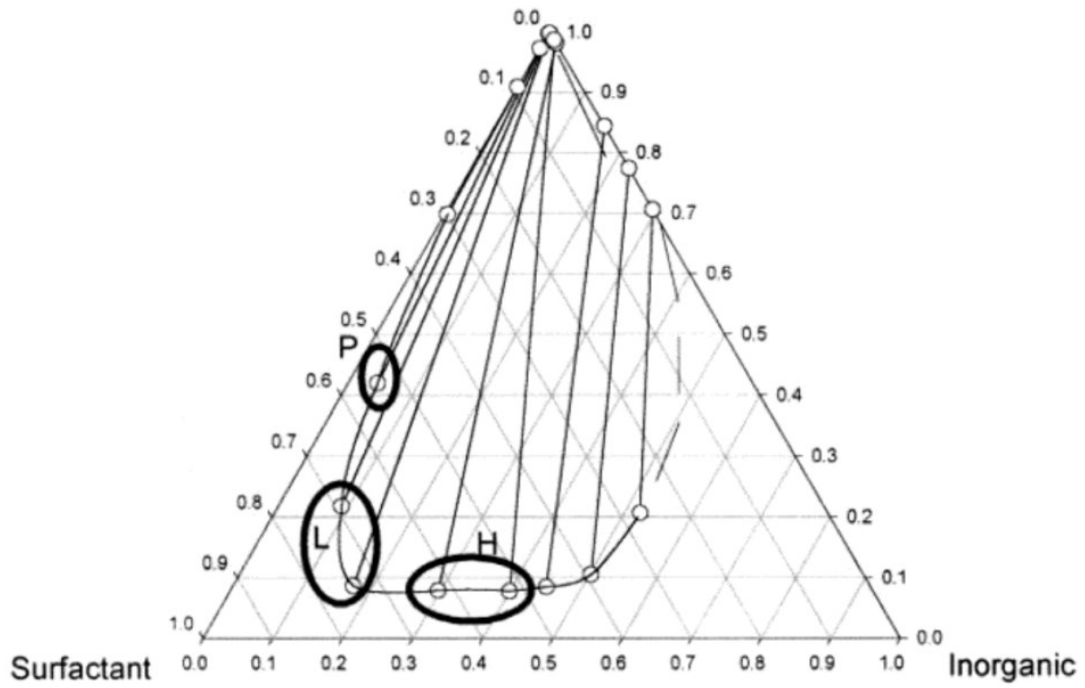

b)

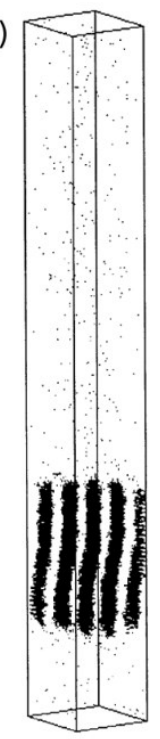

c)

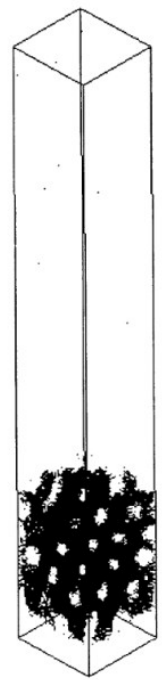

Figure 1. a) Ternary phase diagram of surfactant/water/silica obtained from lattice Monte Carlo simulations, showing the phase separation region. Circles highlight different mesostructures formed in the concentrated phase: $H$ - hexagonal; $L$ lamellar; $P$ - perforated lamellae. Simulation snapshots showing examples of the lamellar phase (b) and of the hexagonal phase (c). Only the silica beads are shown for clarity of visualisation. Adapted from Siperstein and Gubbins [39].

In the ternary systems (surfactant-solvent-silica) Siperstein and Gubbins considered two different cases: in the first, silica was completely soluble in the solvent 
(zero I-S interaction energy), and in the second, it was immiscible (positive I-S interaction). They linked these to two possible mechanisms for inducing phase separation, respectively associative and segregative separation. In the former, the separation is driven by strong attractions between two species, while in the latter it is driven by a strong repulsion. They showed that, independent of the type of mechanism, at high silica/surfactant ratios hexagonal phases (Fig. 1b) were formed, while lamellar phases (Fig. 1c) were formed at low ratios. This corresponds to what is observed experimentally during the synthesis of PMS materials $[2,27]$. However, they were only able to capture qualitative trends, due to the inherent simplicity of the lattice model that restricts the number of surfactants that can interact with a silica species at any given time.

Finally, they investigated the dependence of temperature and surfactant composition on the phase diagrams. They noted that increased temperature led to lower surfactant concentrations but higher silica concentrations in the surfactant-rich phase, which promoted the formation of hexagonal as opposed to lamellar phases. This behaviour can be seen experimentally in CTAB-silica-water systems [23]. Their analysis of surfactant composition showed that increased head/tail ratios (i.e. shorter hydrophobic tails) promoted hexagonal phases, whereas decreased $\mathrm{H} / \mathrm{T}$ ratios favoured lamellar phases.

The model of Siperstein and Gubbins has been subsequently extended to describe phase equilibrium of periodic mesoporous organosilica percursors [41-44], which is discussed in section 4 , and of block copolymer-templated materials [45, 46], which is discussed in section 3 , as well as to explicitly describe silica condensation reactions during the formation of MCM-41 by Jin et al. [47]. The model of Jin et al. introduced three main improvements over the Siperstein model in an effort to increase 
its realism: i) silicates were only allowed to occupy sites on a diamond sublattice of the overall system, thus describing their tendency to form tetrahedral network structures; ii) polymerisation of silica was accounted for by allowing two oxygens to occupy the same lattice site with a very favourable interaction energy (an order of magnitude larger than the silica-head interaction); iii) deprotonation equilibrium was explicitly modelled through a Reactive Monte Carlo (RMC) method, thus allowing for simulations at high $\mathrm{pH}$ (i.e., containing anionic silicates) to be performed. The energy scale was calibrated by attempting to replicate the high-temperature region of the experimental phase diagram of CTAB in water.

Jin et al. considered two different scenarios to attempt to elucidate the synthesis process of MCM-41 [47]. The first scenario was a two-step process: in the first stage no silica polymerisation took place and the system was allowed to self-assemble and phase separate, while in the second stage silica was allowed to polymerise. In the first stage, the model predicted that a binary surfactant/solvent system formed spherical micelles, whereas adding a significant concentration of silica caused the system to form hexagonal arrays of pores, i.e. an MCM-41 template (Fig. 2a). This is in agreement with the theory that cooperative templating is the dominant mechanism during MCM-41 synthesis. They also showed that the initial mesophase formation process is reversible, by cooling and heating the system to move back and forth between the lamellar and hexagonal mesophases. However, this reversibility was only possible before extensive silica polymerisation took place; after polymerisation (second stage), the structure became locked in. 


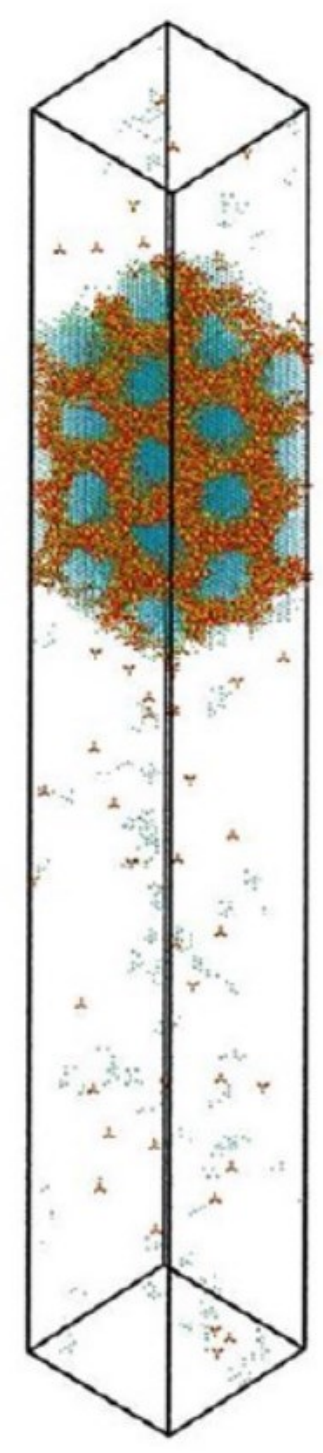

a)

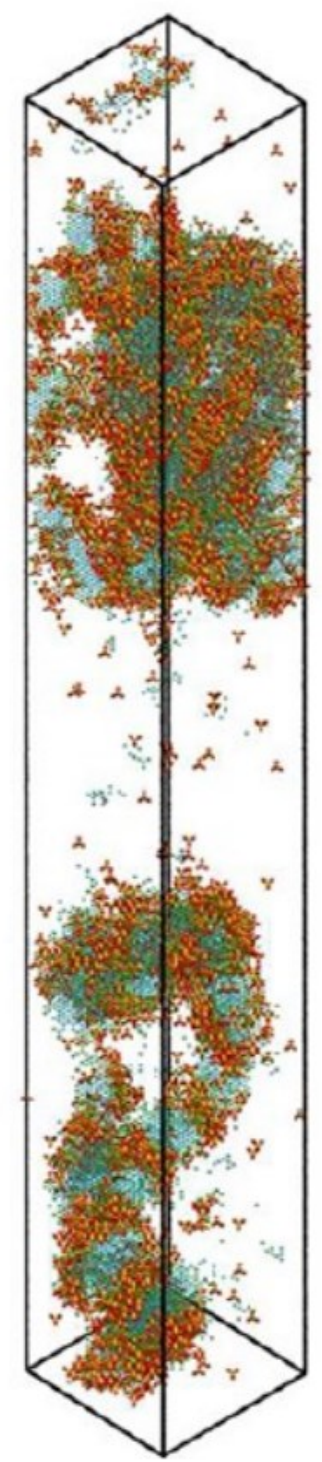

b)

Figure 2. Snapshots of mesophases obtained from lattice Monte Carlo simulations accounting for both surfactant self-assembly and silica polymerisation: a) hexagonal phase obtained from a two-step process; $b$ ) disordered phase obtained from a one-step process. Colour code is: blue - surfactant tails; green - surfactant heads; red-neutral oxygens; purple-negatively charged oxygens; yellow - silicon. Adapted from Jin et al. [47].

The above results were compared to a second scenario in which both mesostructure self-assembly and silica condensation were allowed to take place simultaneously from the very start of the simulation. Interestingly, they observed that rapid polymerisation precluded development of long-range order of the template, 
instead producing a glassy material (Fig. 2b). This result suggests that the mesoscale structure of the final solid can be controlled only during the initial stages of the synthesis, before silica condensation becomes extensive. Even though the results of this study were very insightful, the inherent simplicity of the lattice model once again makes it difficult to establish quantitative comparisons with experiment. For example, the authors were not able to achieve the same degree of silica polymerisation in their simulations as observed in experiments, a discrepancy that was attributed by the authors to geometric restrictions of the lattice [47].

\subsection{Self-Assembly - Off-Lattice Models}

In 2009, Jorge et al. published the first molecular dynamics (MD) investigation of MCM-41 synthesis using realistic atomistic models [48]. They started from a dilute aqueous solution of surfactant, to which anionic silicic acid monomers were added, in an attempt to mimic the initial stages of the synthesis of MCM-41 at high $\mathrm{pH}$ and low surfactant concentrations (the conditions of the original experimental work [2]). Decyltrimethylammonium bromide (DeTAB) was chosen as a surfactant instead of the more common CTAB for the sake of computational tractability, and was modelled using a modification of the OPLS potential [49]. Water was described by the SPC/E model [50]. To describe silicate molecules, Jorge et al. adapted an existing model from Pereira

et al. [51], with modified geometric and electrostatic parameters obtained from quantum calculations on a wide range of neutral and anionic silicates [52].

Their initial study [48] yielded two major insights into the early stages of the synthesis: i) silicates were observed to interact strongly with small surfactant micelles, rather than with free surfactant monomers; ii) adsorption of silica monomers on the 
micelle surface caused an increase in the size of the micelles. This was followed by a more detailed report, in which solutions of silicate oligomers were also considered [53]. More precisely, the authors ran simulations from several starting configurations, whose composition each corresponded to a different stage of the synthesis: reference solution (no silicates present, describing the initial surfactant solution), monomeric solution (with silica monomers instead of bromide counterions), and oligomeric solution (with small silica oligomers corresponding to a distribution inferred from solution NMR experiments at high $\mathrm{pH}$ [54]). From their results, they concluded that cooperative templating does indeed occur, since the size of surfactant micelles was greater in the monomeric solution (Fig. 3b) than in the reference solution (Fig. 3a), and the oligomeric solution produced even larger micelles. At the $\mathrm{pH}$ being modelled (11) almost all the silica species were deprotonated, and it was thus concluded that the increase in micelle size was due to silica species being more effective at screening the positive charge of the surfactants than bromide ions. This was quantified by the average number of surfactants bound to each counterion: 1.31 for bromide ions (reference solution) and 1.64 for silica monomers (monomeric solution). The oligomers were even more effective at producing larger micelles since they can be multiply charged, increasing their screening ability. Interestingly, the multiply charged silica oligomers were also able to bind to multiple micelles simultaneously (1.36 on average, compared to a little more than 1.0 for both bromide and monomeric ions), which was suggested to promote aggregation of micelles. 

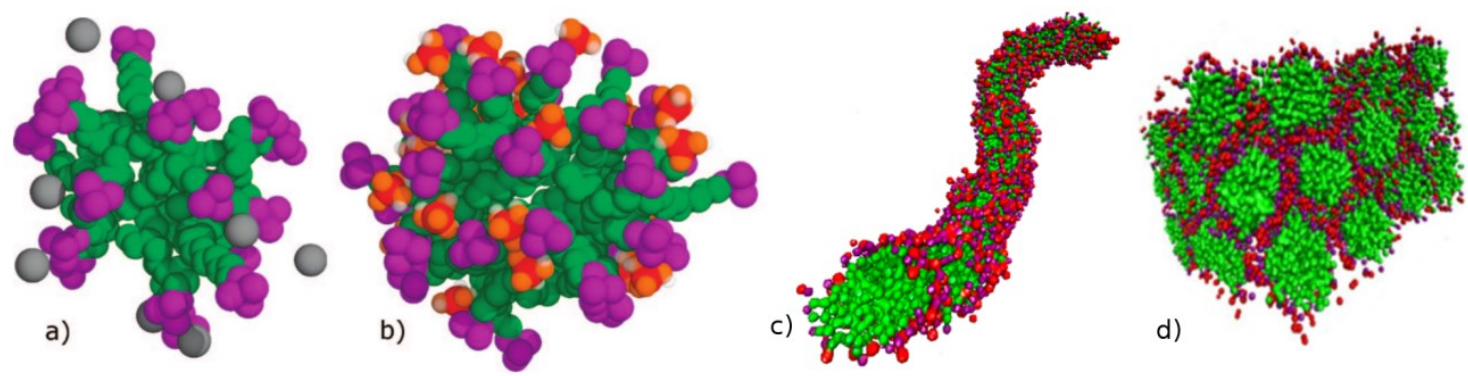

Figure 3. Snapshots showing the evolution of silica/surfactant aggregate structures during the early stages of MCM-41 synthesis, as observed from MD simulations using a multi-scale modelling approach: a) small spherical micelle in a dilute surfactant/water solution (atomistic model), adapted from Jorge et al. [53]; b) larger spherical micelle in a solution with silica monomers at short times (atomistic model), adapted from Jorge et al. [53]; c) rod-like micelle in a silica monomer solution after long equilibration times (coarse-grained model), adapted from Pérez-Sánchez et al. [55]; d) hexagonal phase in a solution with silica dimers (coarse-grained model), adapted from PérezSánchez et al. [60]. Surfactant tails are shown in green, heads in purple, silica in red/orange and bromide in grey.

Jorge et al. [53] also considered an exchange solution, which was produced by taking an equilibrated reference solution and adding silica monomers, while keeping the initial concentration of bromide counterions. This was meant to more realistically mimic the effects of addition of a silica source to a pre-equilibrated surfactant solution. Results of that simulation confirmed that silica preferentially binds to the micelles, and clearly showed ion exchange between silica and bromide at the micelle surface (the degree of silica binding was $76 \%$ by the end of the simulation). The silica monomers tend to be found inside the layer of the head groups, whereas the bromide ions tend to be located outside, allowing the two to work together to more efficiently screen the electrostatic repulsion between head groups, leading to larger micelles. Interestingly, when silica monomers were replaced by larger silica oligomers (keeping the total charge constant), the latter tended to migrate out of the head group layers, facilitating the binding to multiple micelles.

The approach of Jorge and co-workers suffered from several limitations imposed by the computationally intensive nature of the atomistic model. For example, formation 
of multiple micellar aggregates within a reasonable computational time was only possible for the shorter DeTAB surfactant, and not for the more commonly employed CTAB. More importantly, the authors were not able to observe significant shape changes of the micelles (e.g., sphere-to-rod transitions) in their simulations. In an attempt to circumvent those limitations, the same group developed a coarse-grained (CG) model for PMS precursor solutions [55]. The model of Pérez-Sánchez et al. was based on the popular MARTINI CG potential of Marrink and co-workers [56], but included new parameters for neutral and anionic silicic acid monomers. The CTAB surfactant was described by four hydrophobic tail beads and one positively charged head bead [57], while the models for water and bromide followed the conventional MARTINI prescription [56]. The surfactant model was validated by comparing micelle density profiles at the CG [55] and all-atom (AA) levels [58], and was shown to quantitatively reproduce the experimental average size and aggregation number of small CTAB micelles [55], as well as most features of the experimental CTAB/water phase diagram over a wide range of concentrations and temperatures [59]. The silica monomer parameters were calibrated by matching micelle density profiles against those obtained from AA simulations [48] under the same conditions.

Applying the CG mapping procedure allowed Pérez-Sánchez et al. to significantly extend the length and time scales available to MD models of the MCM-41 synthesis (simulations reached the order of tens of microseconds), while maintaining a good degree of chemical realism [55]. Simulations with silica monomers equilibrated to form long wormlike micelles (Fig. 3c), while at the same conditions an aqueous solution of CTAB (i.e., without silica) yielded only a distribution of small spherical micelles. They indeed observed a sphere-to-rod transition in the silica/surfactant solution that proceeded through the successive fusion of several smaller micelles to form large rods. 
This transition was driven by a strong adsorption of anionic silicates on the micelle surface, screening the repulsive interaction between cationic surfactant heads and promoting the formation of structures with lower curvature. This is further evidence supporting the cooperative templating mechanism for the initial stages of MCM-41 synthesis.

In a later paper, Pérez-Sánchez et al. extended the model to consider silicate oligomers, as well as monomers, with parameters once more calibrated to match atomistic micelle density profiles [60]. They focused their attention on low surfactant concentration solutions corresponding to the experimental studies of Firouzi et al. [23]. Initially, they tested several compositions in order to determine the effect of different silica species on the final mesostructure. These systems were: 1) a binary surfactantsolvent reference solution; 2) a ternary surfactant-silica monomer-solvent solution; 3) a ternary surfactant-silica dimer-solvent solution. The silica/surfactant ratio was 1 in the last two systems, i.e. there were half as many dimers in solution 3 as monomers in solution 2; because monomers all had a charge of -1 and dimers of -2 , the total silica charge was kept constant. Their results confirmed the previous assertion [55] that the reference system produced spherical micelles, whereas the introduction of silica altered the geometry of the micelles, making them much more elongated. Interestingly, however, the solutions containing monomers and dimers did not produce the same micellar geometry; solution 2 produced wormlike micelles, whereas in solution 3 a phase-separated hexagonal array, strongly reminiscent of MCM-41 structures, was obtained (Fig. 3d). In order to show that these configurations were equilibrium states, as opposed to kinetic artefacts, exchange simulations were carried out; monomers were replaced by dimers and vice versa, while maintaining the micellar geometry and allowing the system to relax. These simulations indeed showed that the changes were 
reversible, and the obtained structures thus corresponded to equilibrium states of the system.

The hexagonal array reached equilibrium much quicker in the exchange solution than from a random initial configuration. This is interesting, since the exchange solution is likely to represent a more realistic scenario; silica monomers initially produce rodlike micelles, then dimers resulting from silica condensation subsequently promote the formation of the hexagonal array. Further simulations with mixtures of monomers and dimers showed that formation of the hexagonal mesophase requires the presence of between $15-33 \%$ of silicates in the form of dimers. Their results thus not only demonstrate that silica is necessary to promote the formation of the hexagonal structure, but that it needs to be in the form of (at least) small oligomers. This is mainly due to the ability of multiply charged silica oligomers to bind to more than one micelle at the same time, thus promoting micelle aggregation and eventually phase separation of the mesophase.

Next, Pérez-Sánchez et al. investigated larger oligomers, namely cyclic trimers, cyclic tetramers and cubic octamers. Their simulations showed reversible hexagonal to lamellar transitions by changing the silica charge density (either through increasing the degree of condensation while keeping the charge constant, or through increasing the degree of deprotonation for the same degree of condensation) or by adding a co-solvent (e.g. benzene) to the system. In the former case, a higher charge density reduced the effective area per head group leading to a decreased curvature and favouring lamellar phases. In the latter case, the co-solvent was incorporated into the hydrophobic region of the hexagonal mesophase, leading to an increase in the effective tail volume, and hence also promoting a transition to a lamellar structure. All of the above results were in qualitative agreement with experimental observations in the synthesis of MCM-41, but 
a key achievement was the ability to reproduce, for the first time, the formation of a hexagonal silica/surfactant mesophase under conditions that were quantitatively equivalent to the experiments of Firouzi et al. [23].

A very recent paper by the same authors applied the CG model to precursor solutions spanning a wide range of surfactant concentrations, in order to probe the whole phase diagram of the system (Fig. 4) [59]. Once again, they compared the behaviour of a reference $\mathrm{CTAB}$ solution to that of solutions containing silica monomers and silica dimers. For the monomer-surfactant-solvent system, they found no surfactant concentration at which a hexagonal phase could be induced - in fact, the silica monomers appeared to hinder the formation of a hexagonal phase, instead reverting to either wormlike micelles or lamellar phases. Replacing the monomers with dimers produced very different results - a hexagonal phase was formed over a much larger surfactant concentration range than in the binary surfactant-solvent system (mainly at the expense of the micellar rods region). Interestingly, the results suggest that even when starting from a pre-equilibrated hexagonal CTAB structure formed in concentrated surfactant/water solutions, the original surfactant liquid-crystal is destroyed in the initial stages of the synthesis, upon addition of silica monomers, and later reformed after some degree of condensation produces oligomers. These results are further evidence against the LCT mechanism, since they show that the nature of the surfactant-water and of the silica-surfactant hexagonal phases is remarkably different - the former is driven by micelle repulsion (week screening limit) and fills the entire space, while the latter is driven by micelle attraction (strong screening limit) and constitutes a phase-separated system. 


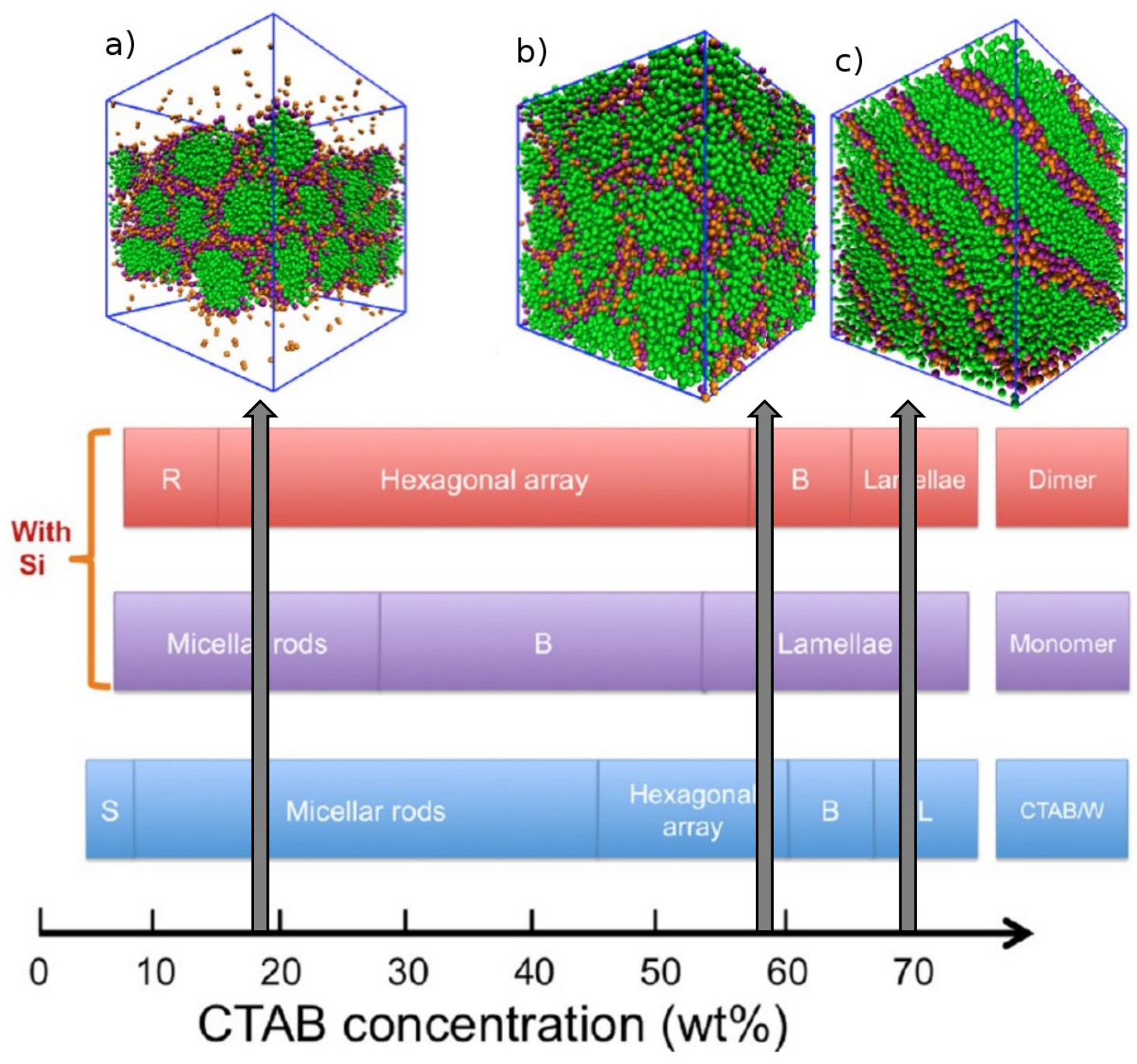

Figure 4. Map of the mesophases observed in coarse-grained simulations of different solutions at a range of initial surfactant concentrations, representative of incremental stages of PMS synthesis, from bottom to top: surfactant/water solution; silica monomer solution; silica dimer solution. $S$ stands for spherical micelles, $R$ for micellar rods, $B$ for bicontinuous phase, and $L$ for lamellar phase. The grey arrows represent possible synthesis paths leading to different PMS materials: a) MCM-41; b) MCM-48; c) MCM50. Adapted from Chien et al. [59].

The phase diagrams for the three systems also allowed the authors to hypothesise on which of the MCM-like materials might be synthesised through a LCT mechanism. At high surfactant concentrations in the presence of silica, they observed the formation of a bicontinuous phase reminiscent of MCM-48 and of a lamellar phase resembling MCM-50. Although bicontinuous phases were observed in all three tested solutions, their ranges of stability did not overlap at any surfactant concentration, hence 
MCM-48 would not seem to be a candidate for the LCT mechanism. The exception, however, is MCM-50; in all three systems, there is a region of overlap of lamellar phases at high surfactant concentrations. Taking all these observations together, it seems likely that the CTM is the main mechanism by which the synthesis of MCM-41 is achieved.

\subsection{Modelling Silica Condensation}

The studies mentioned until this point have all focussed on the self-assembly and phase equilibrium aspects of the PMS synthesis. To investigate those phenomena thoroughly and efficiently, most studies have foregone investigation of the silica polymerisation component of the synthesis - in fact, of all the studies discussed in sections 2.1 and 2.2, only the paper of Jin et al. [47] explicitly described silica condensation reactions, albeit with a rather simplified approach. However, in parallel with that stream of investigation, researchers have been developing increasingly accurate and efficient models and algorithms to describe chemical reactions involving silicates, with several different approaches being proposed. These different approaches, including their advantages and limitations, have been covered in recent reviews $[16,61]$ so we will mention them only briefly here and focus in more detail on the few existing studies that attempted to explicitly include silica condensation reactions in simulations of PMS formation.

Most attempts to describe silica condensation have made use of MD simulations with reactive potentials [62-64]. Such studies were able to provide atomic-level detail on the formation of small amorphous silicate clusters in solution, but required simulations to be run at very high temperatures in order to sample the reaction events. 
The work of $\mathrm{Wu}$ and Deem [65] also used a reactive potential, but the configurational space of the system was explored through a rather complicated Monte Carlo (MC) algorithm. Although their work yielded important insight into silica nucleation, it was limited to small clusters due to the computationally intensive nature of the method. A very distinct approach is kinetic Monte Carlo (KMC) [66-68], which describes chemical reactions as independent Poisson processes and thus significantly speeds up the sampling of reactive events. However, the method requires all reactions and rate constants to be explicitly enumerated, which itself imposes a limitation on the size of oligomers that can be formed (the number of possible reactions increases factorially with cluster size). Models that describe the system energy as a function only of bond topology, such as the Continuous Random Network (CRN) [69, 70], have also been proposed. Finally, recent work by Malani et al. $[71,72]$ has put forth a reactive Monte Carlo (RMC) model of silica polymerisation, which showed good agreement with experimental NMR measurements of the evolution of silicate distributions under neutral conditions. The RMC approach seems promising, as it is able to sample long time scales while maintaining a reasonable level of atomic detail.

To our knowledge, none of the methods described above has yet been applied in the context of simulating the synthesis of PMS materials, with one notable exception being the work of Seaton and colleagues $[73,74]$. The first model that introduced explicit silica polymerisation during simulations of MCM-41 formation was presented by Schumacher et al. [73]. They implemented a KMC reaction scheme, based on the CRN model of the silica network [70] where all silicon atoms are always connected to four oxygens, and tried to describe the entire process of MCM-41 formation. They split their simulations into several stages: i) initial adsorption and condensation of a thin silica layer around the surfactant micelle; ii) aggregation of silica-covered micelles to 
form a hexagonal mesostructure; iii) further condensation of silica to form a highly cross-linked network; iv) high-temperature calcination, causing further silica condensation in the absence of template micelles. To make the scheme computationally tractable many major assumptions were made, such as describing the surfactant micelles as soft cylinders with no atomistic detail. Furthermore, several non-physical moves were applied to force the reaction along the chosen path (e.g. adding an artificial potential gradient to force the silica to the micelle in the opening stages, which is then removed, instead of correctly describing silica diffusion to the micelle surface). Due to all these special moves and assumptions, one cannot be completely confident that this model produced an accurate description of the real synthesis process. However, it achieved the authors' main objective, which was to generate realistic models of MCM41 materials (Fig. 5a) for adsorption predictions (they reproduced gas adsorption fairly accurately for nitrogen, ethane and carbon dioxide). More importantly from the point of view of this review, the work of Schumacher et al. demonstrated that by applying clever approaches, the computational modelling of the entire PMS synthesis process is not an impossible task. 

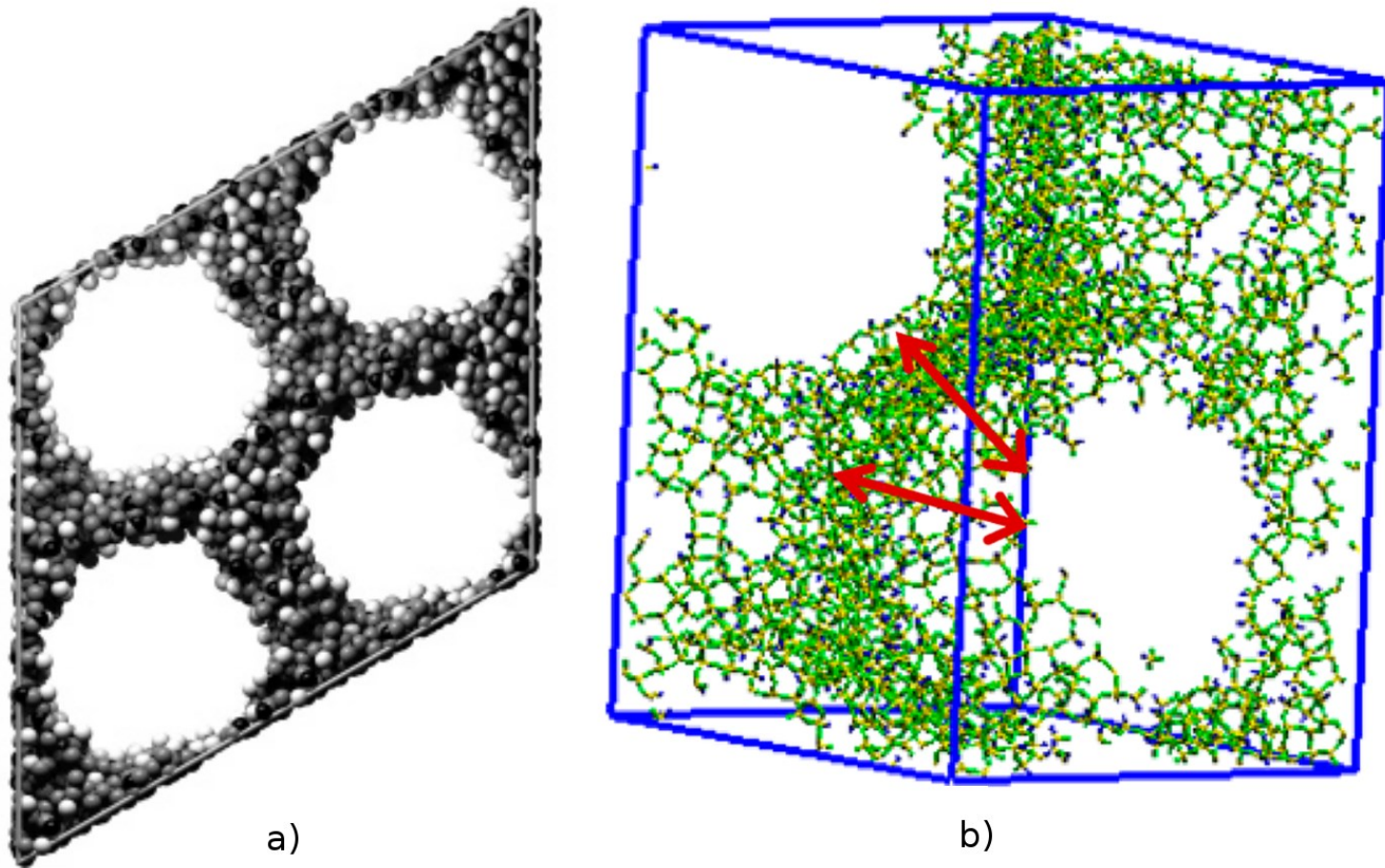

b)

Figure 5. Examples of atomically detailed models of MCM-41 (a) and SBA-2 (b) materials obtained from kinetic Monte Carlo simulations of silica condensation. Only the silica framework is shown for clarity. In panel b), the red arrows highlight connecting windows between the spherical-like pores. Adapted from Schumacher et al. [73] (a) and Ferreiro-Rangel et al. [74] (b).

A later study by Ferreiro-Rangel et al. [74] proposed some improvements to the method of Schumacher and applied it to the synthesis of two less-studied materials, SBA-2 [75] and STAC-1 [76]. Both are synthesized with gemini quaternary ammonium surfactants and contain connected spherical pores templated by spherical micelles, but they differ in the way that the micelles pack together - SBA-2 shows a hexagonal close packing of spheres, while STAC-1 micelles pack in a face-centred cubic lattice. In order to produce realistic models of these materials, Ferreiro-Rangel et al. [74] had to explicitly include water molecules during the reaction stages (which had been ignored by Schumacher et al. [73]) and make the micelle-micelle interactions more repulsive so as to generate the correct packing. The simulation results were successfully compared against experimental data, including structural characteristics and nitrogen adsorption isotherms. The authors concluded that the strong adsorption sites observed in 
experimental isotherms were actually due to surface roughness of the pores, and also suggested a plausible hypothesis for the origin of the connecting windows between large spherical pores (Fig. 5b) during the synthesis [74]. Another interesting observation pertains to the evolution of the degree of silica condensation during different stages of the synthesis. The results of Ferreiro-Rangel et al. show that the transition between the formation of a silica layer at the micelle surface (step 1 of their process) and the initial aggregation of micelles to form an ordered mesostructure (step 2) involved a significant presence of dimers and higher silica oligomers, with the concentration of silica monomers staying below $10 \%$. This is in qualitative agreement with the interpretation of Pérez-Sánchez et al. [60] in their MD study of the self-assembly of silica/surfactant mesostructures.

\section{Synthesis of other mesoporous silica materials}

The synthesis process of the MCM family of mesoporous materials has been the most widely studied so far, particularly from the modelling point of view. However, as research into periodic mesoporous silica progressed, new materials were synthesised by varying the type of template beyond the "traditional" quaternary ammonium surfactants. The latter have small heads with permanent net positive charge and alkyl chains of varying length (normally between 10-18 carbon atoms). By substituting these cationic

surfactants for anionic or non-ionic surfactants and much larger block copolymers, researchers were able to synthesise PMS materials with densities, pore geometries, and structures differing from MCM types [7-10, 77-80]. Despite conceptual similarities, there are important specificities in the synthesis mechanism of such materials. For example, block copolymer templates are usually neutral, and this has implications on the dominant interactions in the system [7]. Amine surfactants, on the other hand, are 
ionisable, and so respond to changes in $\mathrm{pH}$ of the solution during the synthesis process [9]. In this section, we review recent attempts to shed light on the synthesis process of these fascinating materials through computational modelling.

\subsection{Block copolymer templated materials}

Although several early studies managed to synthesise mesoporous silica materials using non-ionic surfactants [77, 78], it was the synthesis of hexagonally ordered SBA-15 (SBA stands for Santa Barbara Amorphous) that represented a major breakthrough in the field [8]. This material was synthesised using non-ionic triblock copolymers as supramolecular templates, and achieved much larger pore sizes (up to 30 $\mathrm{nm})$ than had hitherto been realized. The block copolymer templates, often referred to as Pluronics, consist of portions of poly(ethylene oxide), PEO, and poly(propylene oxide), PPO, arranged as PEOn-PPOm-PEOn. The extra methyl group in PPO makes these moieties more hydrophobic than the linear PEO, and promotes self-assembly of the polymer into large micelles and, at higher concentrations, liquid-crystal mesophases [81]. SBA-15 in particular is synthesised by the sol-gel method in acidic medium by mixing Pluronic P123 $\left(\mathrm{PEO}_{20}-\mathrm{PPO}_{70}-\mathrm{PEO}_{20}\right)$ with a source of silica, most often TEOS [8]. Similarly to MCM-41, this solution is heated to allow the silica to condense around the template mesostructure, followed by template removal by calcination. Despite similarities in the manufacturing procedure, SBA materials have several important distinctions from MCM-type materials. Apart from possessing larger pores than their MCM counterparts, SBA-15 materials also exhibit thicker walls and, more interestingly, small micropores that interconnect the larger, spherical, mesopores [82]. Furthermore, contrary to the case of ammonium surfactants, where ionic interactions dominate, the self-assembly of Pluronics (and arguably of other non-ionic surfactants) hinges on much 
more delicately balanced hydrophobic interactions and/or hydrogen bonds [81]. This poses additional challenges to both experimental and theoretical approaches aiming to describe the synthesis mechanism of block copolymer-templated materials.

Although experimentally the synthesis mechanism of SBA-15 has received perhaps as much attention as that of MCM-41 [83], simulation studies have been much fewer. This is not least because of the much larger size of the surfactant molecules (Pluronic P123 has about 17 times more atoms than CTAB) and micelles, and concomitantly slower dynamics. This puts it firmly outside the scope of detailed atomistic models, except for analyzing local phenomena such as single-chain dynamics [84]. As a consequence, the few existing studies on modelling the synthesis of SBAtype materials have been carried out using highly coarse-grained models and have almost exclusively focused on SBA-15 [45, 46, 85-88].

In 2005, Bhattacharya and Gubbins [45] modified the lattice model of Siperstein and Gubbins [17, 39] to describe the self-assembly of triblock copolymers in the presence of an inorganic component meant to describe silica. They considered surfactants made up of 5 central hydrophobic $(\mathrm{T})$ beads with 3 hydrophilic $(\mathrm{H})$ beads on each end, i.e. $\mathrm{H}_{3} \mathrm{~T}_{5} \mathrm{H}_{3}$ instead of the original $\mathrm{H}_{4} \mathrm{~T}_{4}$ arrangement. The size and shape of the surfactant was selected to produce a water/surfactant phase diagram that qualitatively agreed with experimental data for Pluronic P123. By adding silica to the system, they once again observed phase separation between a dilute and a surfactantrich phase. Depending on the concentrations, the latter formed bicontinuous, hexagonal or lamellar mesostructures. Interestingly, the hexagonal mesophases that are relevant for the synthesis of SBA-15 were only formed at quite low silica concentrations, which was contrary to the observations of Siperstein and Gubbins in their simulations of MCM-41 phase equilibrium [39]. Subsequently, Bhattacharya et al. used the results of the lattice 
model simulations that formed hexagonal phases to produce realistic models of SBA-15 materials, taking into account the molecular scale roughness of the large cylindrical pores and the presence of interconnecting micropores templated by the hydrophilic corona of P123 [46]. The predicted adsorption isotherms were in qualitative, if not necessarily quantitative, agreement with experimental data, leading the authors to suggest that their model was perhaps too rough at the molecular scale.

Bhattacharya and Gubbins also extended their lattice model to include a fourth component, denoted as "oil" and meant to represent the addition of a co-solvent like trimethylbenzene (TMB) to the synthesis mixture [45]. By exploring the quaternary phase diagram, they were able to show a gradual transition between hexagonal (i.e., SBA-15) mesophases and large-scale structures of disordered aggregates reminiscent of the mesocellular foams observed experimentally by gradually adding TMB to the solution [89]. As in the case of MCM-41, the lattice simulations of Gubbins and coworkers were able to qualitatively explore the physics of the phase equilibrium pertaining to block copolymer-templated materials, but it was not possible to carry out quantitative comparisons with the experimental synthesis mechanism due to the inherent simplifications of the model.

An alternative avenue that has been pursued to study the synthesis of SBA-15 is based on the Mesoscopic Dynamics (MesoDyn) simulation method, which has been widely employed to model self-assembly of polymer systems [90]. The MesoDyn method is based on dynamic mean-field density functional theory and the time evolution of the system is propagated through the integration of a set of functional Langevin equations $[85-87,90]$. It uses a coarse-grained implicit solvent description of polymers that are represented by a Gaussian chain of beads, which interact with each other through pairwise interactions based on the Flory-Huggins model. Chen et al. [85] 
applied this approach in the context of SBA-15 synthesis to analyse the effect of temperature on the material pore size. They made use of a previously determined mapping scheme for Pluronic surfactants [90] to describe the P123 triblock copolymer, and used values of the interaction parameters that depended on temperature. Their simulations were able to show the formation of a distribution of spherical polymer micelles at relatively low concentration (Fig. 6a), and posited that the micelle selfassembly process could be divided into three stages [85]. They also observed that the size of the micelle core, formed of hydrophobic PPO beads, increased with temperature, and they linked this to the observed experimental increase in the pore size of SBA-15 with temperature. However, the comparison was merely qualitative, since Chen et al. simulated a simple aqueous solution of P123 (i.e., without the presence of silica) and were not able to observe the formation of a hexagonal phase or even cylindrical micelles [85].

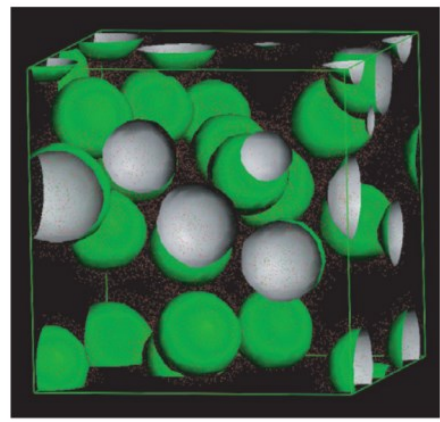

a)

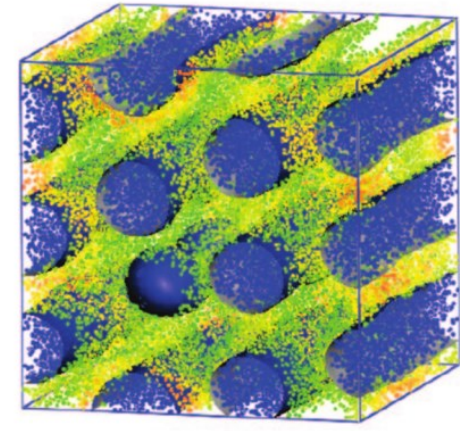

b)

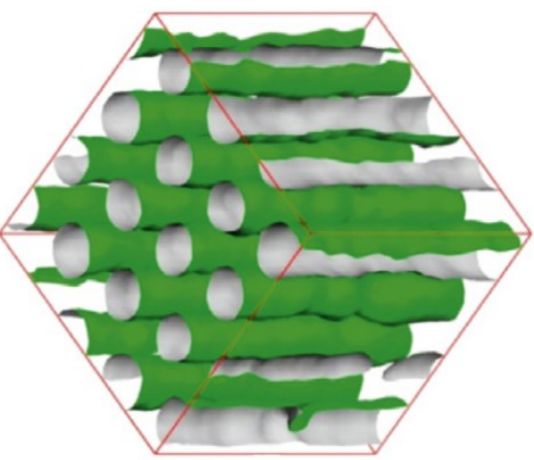

c)

Figure 6. Examples of structures obtained from MesoDyn simulations of Pluronic 123 surfactants: a) spherical micelles obtained in quiescent copolymer/water solutions, adapted from Chen et al. [85]; b) hexagonal mesophase obtained in copolymer/water solutions with constant imposed shear and charged PEO beads, adapted from Yuan et al. [86]; c) hexagonal mesophase obtained in silica/copolymer/water solutions in the presence of shear and with charged PEO beads, adapted from Chen et al. [87].

In the same year, Yuan et al. [86] used a very similar model to study in more detail the self-assembly of P123 at close to room temperature over a wide concentration 
range, and examined the effect of PEO charge and shear on the resulting mesostructures. They observed the formation of several distinct mesophases, including spherical micelles (similar to those described by Chen et al. [85]), bicontinuous and lamellar phases. Interestingly, when a small positive charge was added to the PEO beads (aiming to describe adsorption of $\mathrm{H}^{+}$ions by the micellar corona under highly acidic $\mathrm{pH}$ ) and a constant shear rate was imposed (meant to describe agitation of the solution in experimental SBA-15 synthesis), the authors observed a progressive elongation of the micelles leading to formation of a hexagonal mesophase (Fig. 6b). Interestingly, hexagonal phases seemed to require the presence of both those factors, and were not formed when either shear or PEO charge were separately added to the model [86]. Once again, comparisons with experimental SBA-15 materials were only qualitative, as the authors did not consider the presence of silica in the system.

In 2012, Chen et al. extended their earlier study to include the effect of silica on P123 self-assembly [87]. They reported having obtained the interaction parameters between silica and the other components of the system from "estimating the cohesive energy density of different molecules and the miscibility behavior of binary mixtures" [87], but no additional details were provided. They considered neutral and positively charged (at a fixed value of +1 , meant to reflect protonation under highly acidic conditions [20]) silica beads, as well as varying the charge of the PEO beads. Confirming the observations of Yuan et al. [86], the authors only observed formation of cylindrical micelles when shear was applied to the system. Furthermore, the ordered hexagonal arrangement of the cylindrical micelles (Fig. 6c) was seen to be highly sensitive to the charge values on silica and on the PEO beads - ordered hexagonal mesophases required a charge of +1 on silica (lower or higher values led to disordered micelle packing) and a charge either around -0.3 or around +0.5 on the PEO beads [87]. 
The authors also observed that neutral silica tended to form aggregates, while a relatively uniform distribution of silica around P123 micelles was observed when silica was charged. They attributed this effect to the need for charge matching interactions between silica and surfactant in SBA-15 synthesis [87].

Finally, it is worth mentioning the work of Magee and Siperstein [88], who carried out Dissipative Particle Dynamics (DPD) of ternary systems composed of a diblock copolymer, water, and an inorganic component meant to represent aggregating nanoparticles. DPD is also a mesoscale modelling approach, in which particles interact with each other through soft interactions and with an implicit solvent by way of random (representing Brownian motion) and dissipative (representing friction) forces. Once again, emphasis was placed on mapping the phase diagram of the system. Although the authors were able to observe some interesting physical phenomena, such as the onset of demixing driven by increasing interaction between nanoparticles, it is difficult to draw a direct parallel with realistic experimental systems.

\subsection{Bio-inspired silica materials}

As well as being used at industrial level to produce the classes of porous solids described in the previous sections, templating is also the key mechanism through which many biological organisms produce their complex siliceous structures. This process, referred as biosilicification, is mediated by polypeptides and long-chain polyamines [91], which are believed to catalyse silica deposition while directing structure formation [92], and has attracted particular attention because of its unique characteristics, i.e. it takes place at mild conditions and in an aqueous environment. In fact, just a few years after the discovery of MCM-41, one of the first examples of synthesis of bio-inspired 
silica materials was reported in the literature by Tanev and Pinnavaia [10]. The material, named MSU-V, was obtained using diamines of variable length as templates for silica deposition at ambient temperature and showed a hierarchical lamellar structure [10]. Following this strategy, the same group also employed monoamines to direct structure formation, resulting in the synthesis of a new class of hexagonal mesoporous molecular sieves referred as HMS materials [9, 93]. When compared to their non-bioinspired counterpart (i.e. MCM-41), HMS materials showed as significantly lower degree of order and regularity, which the authors have postulated to be a consequence of the neutral mechanisms behind their synthesis and of the formation of relatively weak hydrogen bonds between template molecules and silicates (as opposed to strong ionic interactions in the case of MCM-41). A similar mechanism of formation primarily based on hydrogen bond interactions between organic and inorganic species was also proposed to describe the formation of MSU-V materials [10]. However, in the case of these diamine-templated silicas the final structure is predicted to arise from silica condensation around multilamellar vesicles, rather than around hexagonally packed surfactant rods. Silica penetration and condensation in the inter-vesicular space gives rise to the formation of pillars that afford structural stability to the material. The relative simplicity of template removal, obtained in the case of bio-inspired materials by means solvent extraction rather than calcination, was also argued to be due to a lack of strong ionic interactions $[9,93]$.

Contrary to cationic ammonium surfactants, amines can be present in aqueous solution in either neutral or protonated forms. This means that these surfactants are $\mathrm{pH}$ responsive, which adds a layer of complexity to the already challenging problem of unravelling the synthesis mechanism of mesoporous silica materials. Recently, multiscale MD simulations have been employed for the first time to shed light on the 
molecular processes taking place during the synthesis of bio-inspired HMS and MSU-V silica materials at varying $\mathrm{pH}[94,95]$. For each bio-inspired material and $\mathrm{pH}$ value considered, two main types of simulation were performed. In a first step, a reference system, containing only surfactant molecules (dodecylamine, DDA, for HMS and 1,12diaminododecane, DADD, for MSU-V) in water was simulated. Subsequently, silicates at different polymerisation degrees were added to represent the early stages of synthesis of these materials. Simulations were carried out at both atomistic (AA) and coarsegrained (CG) level, with results of the former being used to calibrate parameters of the latter. Furthermore, MSU-V synthesis was investigated at ambient temperature, whereas HMS synthesis was studied at a slightly higher temperature $\left(50^{\circ} \mathrm{C}\right)$ to allow comparison with experimental values of micelle aggregation number for the latter system [96].

The multi-scale methodology adopted by Centi and Jorge has two major advantages: it enables to explore longer time and length scales, beyond the current limit of atomistic simulations, while allowing to maintain realism at the lower resolution levels. This involved the development of CG models, based on the MARTINI framework [95] by adjusting the CG parameters until satisfactory agreement between AA and CG density profiles of micellar aggregates was obtained. At the AA level, amine surfactants were described by the OPLS model [97], water by the SPC/E potential [50], and silicates by the model of Jorge et al. [53]. Following the procedure adopted by Pérez-Sánchez et al. to study PMS synthesis [60], the final set of parameters for the systems was obtained in a progressive manner: first, the parameters for the reference system were obtained; subsequently, more species were incrementally added (monomers, followed by dimers) in such a way that interactions already parameterised could be used in more complex systems. Therefore, at each stage only a limited number of unknown interactions had to be determined. Another advantage of this procedure is 
that each step of the model development allows to validate the previously obtained interactions.

CG simulations [95] of the reference systems of HMS materials at experimental concentrations revealed that neutral amine surfactants (i.e. $\mathrm{pH}>12$ ) undergo rapid precipitation, due to their limited solubility in water, which makes it unlikely that they will be involved in the formation of the HMS material. Conversely, charged surfactants $(\mathrm{pH}<8)$ form micellar aggregates with average size in excellent agreement with experimental data [96]. Interestingly, micelles are converted into large branched rods when silica monomers are added to the solution. This result indicates that, contrary to the hypothesised mechanism based on interactions between neutral species [9], charged species are necessary to promote rod formation. Hence, the synthesis of HMS materials was further studied including the effect of more highly condensed silica species, showing that dimers produce a disordered packing of rods in agreement with the "worm-like" or "worm-hole" structures reported experimentally [98], therefore reinforcing the conclusion that charge matching interactions control the formation of HMS materials.

The second type of bio-inspired material considered, MSU-V, was initially studied using AA simulations [94]. Simulations at high $(>11)$ and intermediate (8-11) $\mathrm{pH}$, where the majority of the diamine surfactants is either neutral or singly charged, showed formation of dry layers of surfactants for both systems. However, when silicates were added, strong adsorption of silica monomers at the layer surface only occurred in the system with singly charged surfactants (Fig. 7b), while the monomers remained homogeneously dispersed in the solution with neutral surfactants (Fig. 7a). Conversely, when doubly charged surfactants dominate $(\mathrm{pH}<8)$, formation of small disordered clusters was observed (Fig. 7b), with or without silica. This indicates that formation of 
MSU-V materials is only feasible within the narrow $\mathrm{pH}$ range of 8-11, which agrees with acid-base calculations based on the composition of the experimental solution. Simulations of a more realistic speciation of amines and silicates (based on experimental conditions), in which all three types of surfactants coexist, produced curved layers that suggest a transition to a vesicular-like structure as observed in experiments. Furthermore, the authors observed the spontaneous formation of a hole defect in one of the layers, inside which a few silica monomers, together with water molecules, migrated. It was hypothesised that, due to local excess of positive charges in the surfactant layer, similar holes or defects could be created across template layers allowing more silicates to penetrate and eventually condense inside. This result, therefore, represents strong evidence for the pillaring mechanism postulated experimentally [10]. However, similar to HMS materials, the originally hypothesised neutral templating route does not seem to be feasible; instead, the self-assembly mechanism seems to rely on charge-matching interactions between silicates and amine surfactants. Preliminary CG simulations of MSU-V materials confirm this conclusion [95].

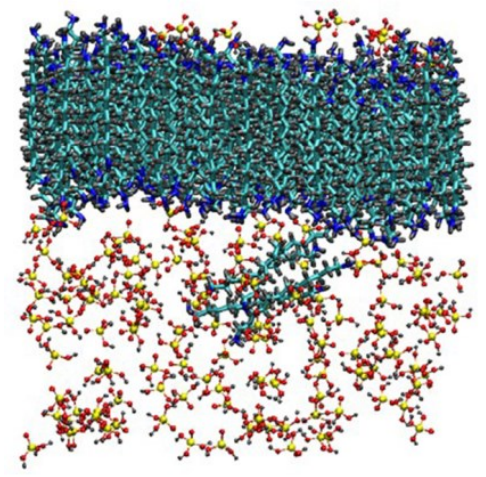

a)

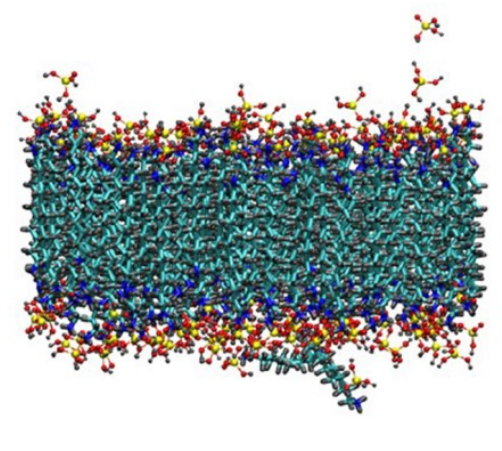

b)

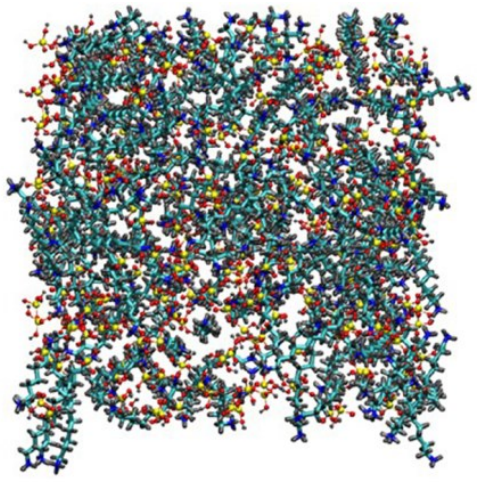

c)

Figure 7. Snapshot of configurations obtained from atomistic MD simulations of MSU$V$ precursor solutions containing silica, water and DADD surfactant: a) high $\mathrm{pH} ; \mathrm{b}$ ) intermediate $\mathrm{pH}$; c) low $\mathrm{pH}$. Colour code is: blue - charged nitrogen; purple - neutral nitrogen; gray - hydrogen; cyan - carbon; red - oxygen; yellow - silicon. Adapted from Centi and Jorge [94]. 


\section{Synthesis of hybrid organosilica materials}

As mentioned above, one of the major breakthroughs in the manufacture of functional mesoporous materials was the direct inclusion of organic groups on the pore walls during the synthesis process (sometimes called "in-situ" functionalisation, in contrast with "post-synthesis" functionalisation, which is not covered in this review), giving rise to periodic mesoporous organosilicas (PMOs) [11-13]. The preparation of these hybrid organic/inorganic silicas from bissilylated organic precursors (i.e., organobridged alkoxysilanes) is also based on surfactant-templated synthesis as in the case of pure silica PMS materials [2], and thus the pore size/shape and wall thickness of the PMO framework can be also controlled by tuning the synthesis conditions $[99,100]$. However, PMOs are even more interesting materials because they combine the strength of the inorganic silica structure with the appealing functionality of organic groups, significantly expanding their range of potential applications [99-102]. As a consequence, the development of these materials has continued to grow, with a promising avenue being the incorporation of ever more complex functional organoalkoxysilane precursors [102-104].

This high degree of variability together with the typically more flexible nature of organic groups brings with it additional challenges from the experimental point of view; for instance, PMO materials tend to be less ordered at the mesoscale level than their allsilica counterparts [102]. It also makes it even more important to obtain a fundamental understanding of the synthesis process, so as to better control the material properties. In this context, the effect of the presence of organic precursors on the synthesis mechanism remains largely unexplored. For example, a particularly interesting discovery was that some aromatic bridging groups tend to form pore walls that possess molecular-scale 
order, i.e. they are semi-crystalline, in contrast with standard PMS materials and other PMOs [105]. It remains unclear what the driving force for the formation of such ordered domains is, or how it can be harnessed in a systematic way. In this context, molecular modelling studies can be very useful to shed light on the dominant variables that control the final structure of the material. As in the synthesis of MCM-41 and related materials (see section 2), computational studies on PMO synthesis have followed two distinct routes: highly coarse-grained lattice models, aiming to probe the phase diagrams of the precursor solutions; atomistic models, aiming to elucidate the intermolecular interactions governing the self-assembly process. These are discussed in the next two subsections.

\subsection{Phase Diagrams - Lattice Models}

Patti et al. [41] first extended the original lattice model of Siperstein and Gubbins [17, 39] to include hybrid organic-inorganic precursors in the synthesis solution. As in the original work, Patti et al. considered linear surfactants, namely $\mathrm{H}_{4} \mathrm{~T}_{4}$. Inorganic silica was mapped as two connected segments on the lattice, with parameters representing complete miscibility $\left(\mathrm{I}_{2}\right)$ or complete immiscibility (I'2) with the solvent (S). The organic groups were considered as either hydrophobic, i.e. equivalent to surfactant tails, or hydrophilic, i.e. equivalent to heads, leading to four distinct possibilities: IT, I'T, IH and I'H. Patti et al. performed MC simulations in the NVT ensemble at different surfactant concentrations and found that the system phase separated into a concentrated surfactant phase and a solvent-rich phase. Interestingly, they found that the nature of the organic group had a profound influence on the formation of ordered mesophases, with hydrophobic groups acting as co-surfactants and hydrophilic groups playing the role of co-solvent. In fact, considering a hydrophobic 
terminal group, $\mathrm{H}_{4} \mathrm{~T}_{4}$-IT-S, no ordered phases were found, even at high surfactant concentrations; however, a hexagonal phase was found at high surfactant concentrations in the $\mathrm{H}_{4} \mathrm{~T}_{4}-\mathrm{IH}-\mathrm{S}$ system [41]. The authors proposed this as a possible explanation for the difficulty in obtaining ordered materials with terminal organic-inorganic precursors [102]. In all ordered mesophases, Patti et al. observed that the terminal organic group was mainly located on the solvent side of the micellar aggregates, thus suggesting that at least some of the organic groups would not be available on the pore surface after the synthesis [41].

A similar approach was later used [42] to study the phase diagrams of systems containing bridging organic precursors (i.e., IHI, I'HI', ITI, and I'TI'), and comparing them to the purely inorganic and terminal hybrid precursors. Once more, they observed phase separation in their systems, driven by the presence of the inorganic components, yielding in some cases ordered mesophases. The main conclusion of their second paper was that bridging precursors are more effective at promoting phase separation and emergence of order in the concentrated phase, presumably because the potentially disrupting organic moieties are "protected" by inorganic groups on both sides.

In a later study [43], the same group extended their approach to consider more complex hybrid precursors, aiming to represent organosilicates functionalised with amine groups, namely: aminopropyl-trimethoxysilane (AP, represented as $\mathrm{I}_{2} \mathrm{TH}$ ), methyl-3-aminopropyl-trimethoxysilane (MAP, described as $\mathrm{I}_{2} \mathrm{THT}$ ) and phenyl-3aminopropyl-trimethoxysilane (PAP, described as $\mathrm{I}_{2} \mathrm{THT}_{3}$ ). They then examined fourcomponent systems comprised of a pure silica source, solvent, surfactant and one of the aforementioned hybrid amino-silicate species. Importantly, their surfactant was no longer linear, as in previous studies, but composed of a branched head (i.e., $\mathrm{T}_{5} \mathrm{HH}_{3}$ ). Patti et al. showed that at high concentrations of AP (15 vol. \%) and MAP (25 vol. \%) 
the hexagonal geometry of the surfactant mesophase broke down, in qualitative agreement with experimental observations. They posited that this was due to the amine groups being relatively solvophilic, leading to the increased solubility of the surfactant in the solvent and promoting formation of disordered phases. Conversely, high concentrations of PAP (above 17 vol. \%) resulted in a transition from a hexagonal to a lamellar phase. In this case, the hydrophobic nature of the large tail group of PAP caused a swelling of the hydrophobic core of the surfactant micelles, which promoted a lower curvature and hence a lamellar phase. In the case of the longer precursors (MAP and PAP), the authors observed that the organic groups penetrated the micelle core, suggesting that they would become available for surface interactions [43]. However, for AP it was shown that the functional amine group was encompassed almost entirely in the inorganic walls, which means that it would be inaccessible as a functional group. The authors point out that this contradicts experimental results, and ascribe the discrepancy to the inherent simplifications of their lattice model.

The last study of this series by Patti et al. [44] considered longer hybrid precursors in the terminal and bridged forms, namely $\mathrm{I}_{2} \mathrm{THT}_{3}$ and $\mathrm{I}_{2} \mathrm{THTI}_{2}$. Similarly to the case with smaller precursors [42], they observed that the bridged arrangement was more favourable for the formation of ordered hexagonal phases. More interestingly, they considered mixtures of the two precursors, leading to a five component system, in an attempt to describe the formation of so-called bifunctional mesoporous organosilicas. Their results showed that the bridging precursor (BP) acted as a co-solvent due to both ends being hydrophilic, whereas the terminal precursor (TP) acted as a co-surfactant. In this way, the surfactant solubility could be controlled by adjusting the concentrations of these two precursor species, and this induced transitions from hexagonal to either cubic or lamellar phases. Where the $\mathrm{BP}$ and $\mathrm{TP}$ concentrations were similar, they tended to 
cancel each other out and a hexagonal phase was formed. However, an excess of the TP relative to the BP produced lamellar phases, and the reverse case produced cubic phases (Fig. 8).

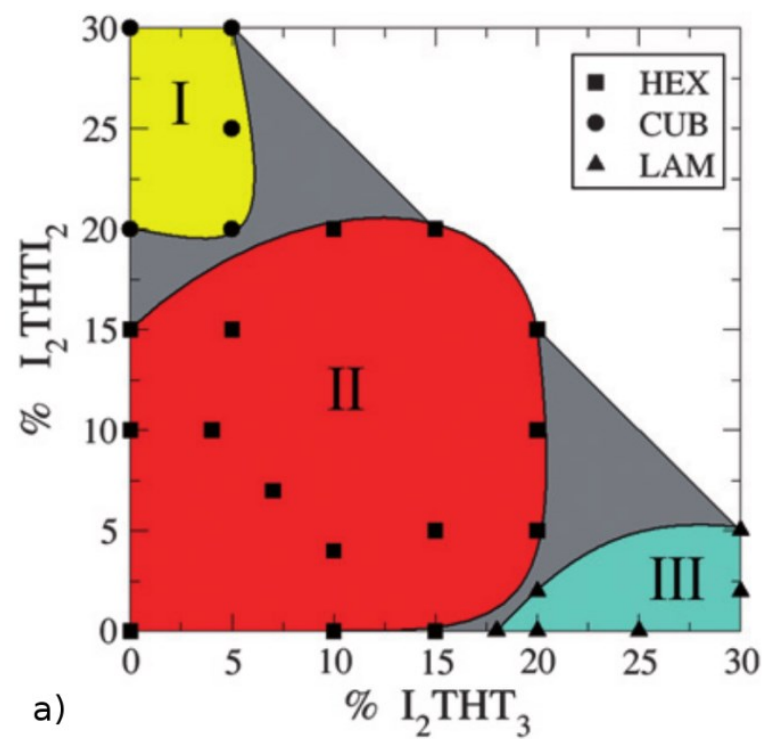

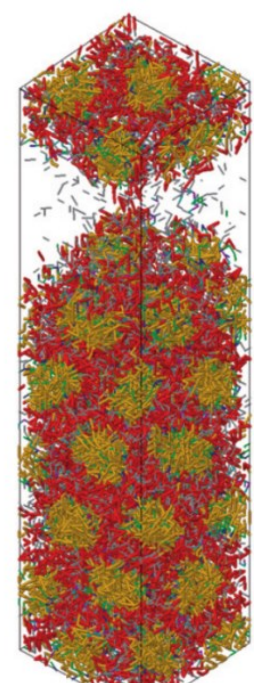

b)

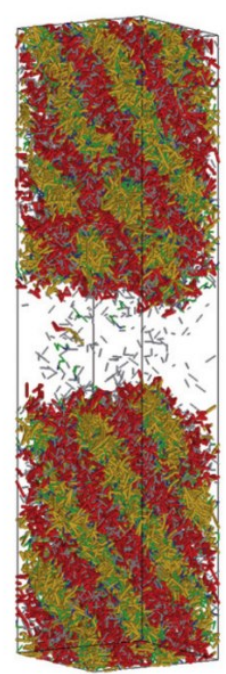

c)

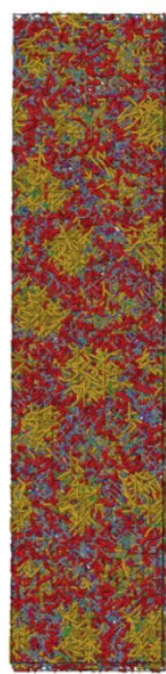

d)

Figure 8. Two-dimensional phase diagram (a) obtained from lattice Monte Carlo simulations of solutions with $40 \%$ surfactant, $10 \%$ silica dimers and variable concentrations of terminal and bridging hybrid organosilicate precursors. The labelled regions indicate stable cubic (I), hexagonal (II) and lamellar (III) mesophases. Snapshots showing examples of hexagonal (b), lamellar (c) and cubic (d) mesophases. Colour code is: red - surfactant heads; yellow - surfactant tails; gray - silica; blue/green - organic groups of terminal precursor; magenta/cyan - organic groups of bridging precursor. Adapted from Patti et al. [44].

\subsection{Self-Assembly-Off-Lattice Models}

Similarly to lattice models, atomistically detailed approaches to study the synthesis of PMO materials have built up from previous work on MCM-41, based on using information obtained at the quantum mechanical level $[52,106]$ to develop allatom models of the synthesis solution $[48,53,107]$ that are able to probe the molecularlevel interactions between precursors and surfactants in systems with several thousands of molecules. Futamura et al. [106, 107] developed an all-atom model for hybrid inorganic-organic precursors $(\mathrm{HO})_{3} \mathrm{Si}-\mathrm{R}-\mathrm{Si}(\mathrm{OH})_{3}$ with different organic linkers $(\mathrm{R})$, 
namely, planar organic ring (benzenesilica, BENZ-Sil), double $\mathrm{C}=\mathrm{C}$ bond (ethylenesilica, ETHY-Sil) and single C-C bond (ethanesilica, ETHA-Sil). The aim was to study the role of the organic linker in the PMO formation, elucidating experimental observations where different organosilica precursors yielded structural differences in the final material [99]. The model was based on a combination of OPLS parameters for the organic groups [97] with silicate parameters from the group's previous work [53] and new geometric and electrostatic parameters, specific to organosilicates, obtained from DFT calculations in the gas phase [106].

Futamura et al. [107] carried out MD simulations of three different aqueous solutions with DeTAB as a surfactant and each of the different organosilicate precursors under high $\mathrm{pH}$ conditions, where the majority of silicate groups were anionic. Their simulations of up to $35 \mathrm{~ns}$ were able to capture the early synthesis stages, showing the initial formation of small surfactant aggregates, followed by micelle growth and micelle fusion processes. The systems with BENZ-Sil and ETHY-Sil showed a measurable increase in the average micelle size with respect to the ETHA-Sil system. This was associated with a higher concentration of precursors adsorbed at the micelle interface for the former two cases, which supports the cooperative templating mechanism, as previously observed in the case of all-silica systems [48].

From computed micelle density profiles, the authors showed that not only the inorganic silica but also the organic part of the precursors could be found between the surfactant heads in the micelle surface [107]. More interestingly, they observed that whereas BENZ-Sil and ETHY-Sil were arranged parallel to the micelle surface, the ETHA-Sil species were placed perpendicularly. This is an important fact because BENZ-Sil and ETHY-Sil are arranged more efficiently in the space between the surfactant heads, making it easier to form ordered structures. Furthermore, a visual 
inspection of the BENZ-Sil system showed evidence of ordered arrangements of organosilicate precursors at the micelle surface (Fig. 9), possibly caused by $\pi$ - $\pi$ stacking between BENZ-Sil species [107]. It was suggested by the authors that this could represent the initial stages of formation of the molecular-scale periodicity experimentally observed in the pore walls of benzene-silica materials [105]. Unfortunately, the high computational expense of the all-atom simulations prevented Futamura et al, from probing later stages of the PMO synthesis, including the formation of mesoscale organosililca/surfactant structures.

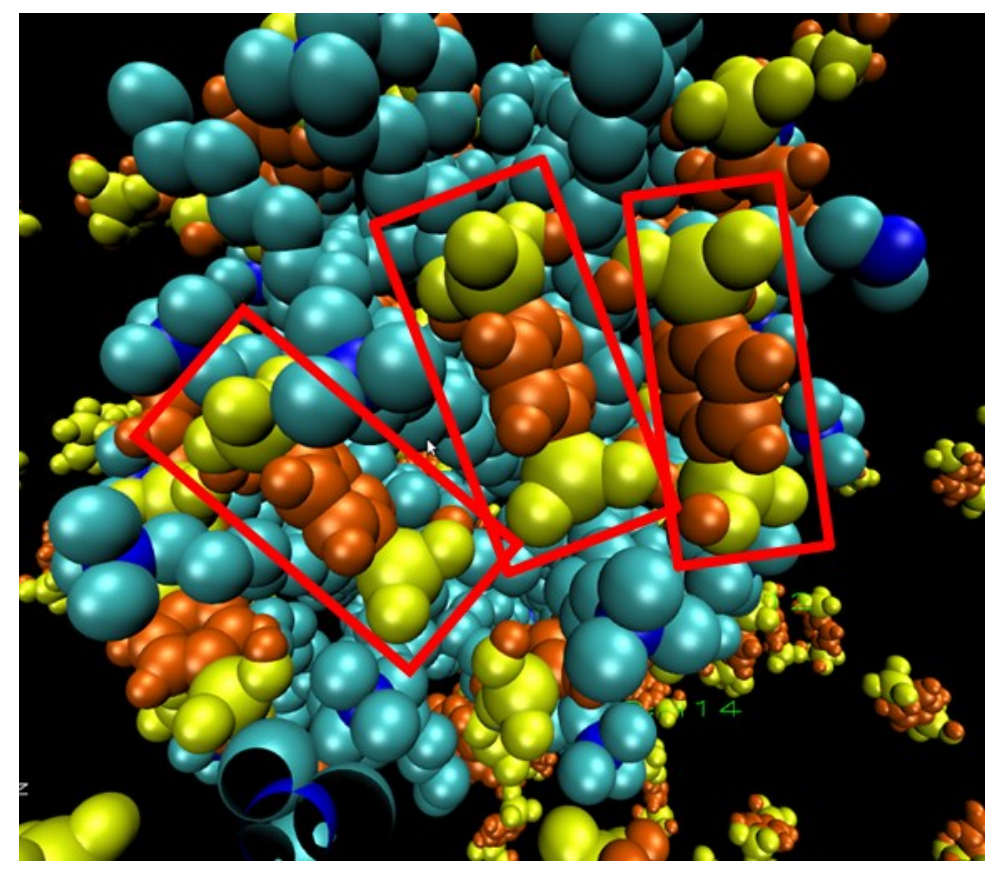

Figure 9. Snapshot of a spherical micelle formed in atomistic MD simulations of a precursor solution of benzene-silica PMO. Colour code is: light blue - surfactant carbon atoms; dark blue - surfactant nitrogens; orange - benzene groups; yellow silicate groups. The red boxes highlight hybrid precursors that appear to stack in an ordered fashion at the micelle surface. Adapted from Futamura et al. [107].

\section{Summary and outlook}

Immense progress has been made in our ability to model the synthesis process of mesoporous silica and organosilica materials, particularly over the last decade. This has followed two distinct lines of attack, which we may call "top-down" and "bottom-up". 
The former is based on describing the underlying physics of the system using highly coarse-grained models, following the principle of Occam's razor, and then probing a relatively wide range of synthesis parameters (concentration, temperature, etc.). Examples of this approach are the lattice model studies of PMS and PMO synthesis [17, 39, 41-47], as well as the MesoDyn simulations of SBA-15 [85-87]. The bottom-up approach, on the other hand, starts at a high level of theory (atomistic or even quantum mechanical), and builds simpler models through a progressive coarse-graining procedure. This strategy has been applied to study the formation of MCM-41 [48, 52, 53, 55, 59, 60], PMOs [106, 107] and bio-inspired silica materials [94, 95].

The synthesis of MCM-41 has predominantly been modelled at high $\mathrm{pH}$ conditions, where the silica precursors are almost all in the anionic form. Modelling studies have revealed that under those conditions, anionic silica monomers replace bromide counterions at the surface of cationic ammonium surfactant micelles, causing an increase in the micelle size through more effective screening of the head group repulsions [53]. This eventually leads to a pronounced shape transition in the micelles, from a predominantly spherical to a rod-like morphology [55]. However, formation of hexagonal structures seems to require the presence of more condensed silicate species, such as dimers and/or small oligomers $[60,74]$, which due to their multiply charged nature can act as "bridges" between adjacent micelles. This changes the balance of interactions, promoting inter-micelle attractions [60] and leading to phase separation into a dilute solution and a concentrated silica/surfactant-rich mesophase through a segregative mechanism [39]. The structure of the mesophase remains labile until extensive silica condensation takes place, thus "locking-in" the structure [47]. Importantly, before such high levels of silica polymerisation are reached, it remains possible to control the properties of the mesophase by changing the synthesis conditions 
(for example, lowering the temperature or adding a co-surfactant to promote a hexagonal-to-lamellar transition) $[47,60]$. The calcination process to remove the template further increases the degree of condensation of the silica network, but does not largely change the nature of the porous structure [73].

Taking theoretical and experimental evidence together, it is clear that MCM-41 synthesis starting from low concentration surfactant solutions takes place through a cooperative templating mechanism via silica/surfactant charge matching, where silicates promote changes in the morphology of surfactant aggregates, while the presence of surfactant micelles creates local concentration gradients that promote silica condensation. However, it appears that even when starting from high surfactant concentration solutions, which are already able to form mesostructures, a true liquidcrystal templating mechanism is unlikely to take place [59]. This is because the addition of anionic silica to the solution causes a profound change in the nature of the molecularlevel interactions, promoting phase separation of an otherwise homogeneous solution. We thus speculate that the LCT mechanism may only be viable in systems that are not dominated by strong electrostatic interactions, such as in materials templated by neutral surfactants [108]. Further simulation and experimental studies are required to verify this assertion.

Comparatively to MCM-41 synthesis at high $\mathrm{pH}$, following the $\mathrm{S}^{+} \mathrm{I}^{-}$mechanism [7], the synthesis of mesoporous materials using anionic or neutral surfactants, or at lower $\mathrm{pH}$ conditions, remains unexplored by modelling. Adaptation of existing lattice models and multi-scale models to consider other mechanisms, including anionic templating and counterion-mediated mechanisms, is thus a natural avenue of research that should be explored in the near future. Systems that involve block copolymer templates are particularly challenging, however, as the much larger template sizes and 
slower dynamics practically preclude atomistically detailed simulations. Despite some attempts at modelling SBA-15 synthesis using highly coarse-grained models $[45,85-$ 87], mechanistic insight has so far been limited, and key questions like the role of silica in the self-assembly process remain unanswered. Additional simulation studies of these systems that are able to establish clearer links between the behaviour of coarse-grained models and realistic experimental systems are urgently needed. This clearly requires a multi-scale modelling approach, perhaps making use of recent developments in block copolymer models [109] and extending them to include silicate species.

Interesting progress has recently been made in the synthesis of mesoporous silica materials templated by amine surfactants. The latter are akin to naturally occurring templates for biosilicification processes, and thus such materials represent the earliest examples of bio-inspired silica. Until recently, the proposed mechanism for synthesis of materials like MSU-V or HMS had been based on indirect experimental evidence and a high degree of speculation. Multi-scale modelling studies have clarified many aspects of the synthesis of these materials [94, 95], showing the self-assembly of structures that strongly resemble those observed experimentally. The main outcome of that work, however, is the demonstration that, contrary to what had been previously hypothesised [9, 10], strong electrostatic interactions between charged species, rather than hydrogen bond interactions, guide the process of formation of these porous solids. Additional simulation and experimental studies are required to confirm or dismiss the viability of the originally proposed neutral templating mechanism. Furthermore, extension of this modelling approach to consider more industrially relevant materials is also desirable. For example, atomistic modelling of interactions between polyamine templates and silica surfaces has recently contributed to the development of more 
environmentally friendly template removal processes in the manufacture of bio-inspired silica [110].

Finally, modelling studies of periodic mesoporous organosilica materials have closely followed those of their "parent" MCM-type counterparts by adding an extra degree of complexity to existing models. Namely, lattice models were extended to a wide range of hybrid precursors [41-44], while atomistic models of such hybrid molecules were also developed $[106,107]$. The lattice models have shown that the organic groups may have a significant effect on the resulting silica/surfactant mesostructures, depending on their hydrophobic or hydrophilic nature [41]. They also showed that bridging precursors are more effective at inducing ordered materials than terminal precursors, thus clarifying experimental observations [44]. Atomistic studies, on the other hand, were able to elucidate the underlying molecular level interactions, concluding that the onset of micro-scale ordering on the pore walls of some PMO materials may take place very early in the synthesis process [107]. Despite these important advances, the picture of the mechanism of PMO synthesis is not as clear as that of MCM-41, partly because of the lack of intermediate-level studies of these systems (i.e., using coarse-grained models that are still able to retain a high degree of chemical realism $[59,60])$. Efforts to develop such a model are already underway at our research group, and we hope to report them in the near future [111].

In methodological terms, the two fundamentally different approaches described above have so far advanced in a largely independent way. Bottom-up approaches have the ability to describe realistic systems at a high level of detail, but are limited to relatively small systems and short time scales. Conversely, top-down approaches can cope with such scales, thus allowing one to potentially probe the synthesis mechanism in its entirety, but the inherent simplifications make it hard to draw direct links to 
experimental materials. Bridging the gap between these two approaches is likely to lead to a paradigm shift in our ability to model PMS synthesis. Recent advances in the synthesis of MCM-41 at high $\mathrm{pH}$ suggest that this in not far from being achieved for the "base case" of PMS synthesis.

A major conceptual advance has been, in our opinion, the ability to simultaneously account for surfactant self-assembly and silica polymerisation within a single simulation [47]. However, this has only been achieved so far for lattice models, which, as we have discussed, suffer from limitations in terms of chemical specificity. Achieving this goal for off-lattice models (atomistic or coarse-grained) is another required methodological leap. The RMC approach of Monson and co-workers [71, 72] appears particularly promising for this purpose, as it strikes a good balance between efficiency and realism. We strongly believe that such improvements in multi-scale, multi-physics modelling will lead to the development of predictive tools that can design materials with optimal properties for specific applications. Although we are still far from this ultimate goal, the future appears bright for this field of research.

\section{References}

[1] Barton, T. J.; Bull, L. M.; Klemperer, W. G.; Loy, D. A.; McEnaney, B.; Misono, M.; Monson, P. A.; Pez, G.; Scherer, G. W.; Vartuli, J. C.; Yaghi, O. M. Tailored porous materials. Chem. Mater., 11 (1999), 2633-2656.

[2] Beck, J. S.; Vartuli, J. C.; Roth, W. J.; Leonowicz, M. E.; Kresge, C. T.; Schmitt, K. D.; Chu, C. T. W.; Olson, D. H.; Sheppard, E. W.; McCullen, S. B.; Higgins, J. B.; Schlenker, J. L. A new family of mesoporous molecular sieves prepared with liquid crystal templates. J. Am. Chem. Soc. 114 (1992), 10834-10843. 
[3] Kresge, C.T.; Leonowicz, M,E.; Roth, W.J.; Vartuli, J.C.; Beck, J.S. Ordered mesoporous molecular sieves synthesized by a liquid-crystal template mechanism. Nature 359 (1992) 710712.

[4] Di Renzo, F.; Cambon, H.; Dutartre, R. A 28-year-old synthesis of micelle-templated mesoporous silica. Micropor. Mater. 10 (1997) 283-286.

[5] Chiola, V.; Ritsko, J.E.; Vanderpool, C.D. Process for producing low-bulk density silica. US Patent (1971) 3556725.

[6] Zhou, H.-C.; Kitagawa, S. Metal-Organic Frameworks (MOFs). Chem. Soc. Rev. 43 (2014) $5415-5418$.

[7] Huo, Q.; Margolese, D. I.; Ciesla, U.; Demuth, D. G.; Feng, P.; Gier, T. E.; Sieger, P.; Firouzi, A.; Chmelka, B. F.; Schüth, F.; Stucky, G. D. Organization of Organic Molecules with Inorganic Molecular Species into Nanocomposite Biphase Arrays. Chem. Mater. 6 (1994) 11761191.

[8] Zhao, D.; Feng, J.; Huo, Q.; Melosh, N.; Fredrickson, G. H.; Chmelka, B. F.; Stucky, G. D. Triblock Copolymer Syntheses of Mesoporous Silica with Periodic 50 to 300 Angstrom Pores. Science 279 (1998) 548-552.

[9] Tanev, P. T.; Pinnavaia, T. J. A Neutral Templating Route to Mesoporous Molecular Sieves. Science 267 (1995) 865-867.

[10] Tanev, P. T.; Pinnavaia, T. J. Biomimetic Templating of Porous Lamellar Silicas by Vesicular Surfactant Assemblies. Science 271 (1996) 1267-1269.

[11] Asefa, T.; MacLachlan, M. J.; Coombs, N.; Ozin, G. A. Periodic mesoporous organosilicas with organic groups inside the channel walls. Nature 402 (1999) 867-871.

[12] Melde, B. J.; Holland, B. T.; Blanford, C. F.; Stein, A. Mesoporous Sieves with Unified Hybrid Inorganic/Organic Frameworks. Chem. Mater. 11 (1999) 3302-3308. 
[13] Inagaki, S.; Guan, S.; Fukushima, Y.; Ohsuna, T.; Terasaki, O. Novel Mesoporous Materials with a Uniform Distribution of Organic Groups and Inorganic Oxide in Their Frameworks. J. Am. Chem. Soc. 121 (1999) 9611-9614.

[14] Ying, J. Y.; Mehnert, C. P.; Wong, M. S. Synthesis and Applications of SupramolecularTemplated Mesoporous Materials. Angew. Chem. Int. Ed. 38 (1999) 56-77.

[15] Slowing, I. I.; Trewyn, B. G.; Giri, S.; Lin, V. S.-Y. Mesoporous Silica Nanoparticles for Drug Delivery and Biosensing Applications. Adv. Funct. Mater. 17 (2007) 1225-1236.

[16] Auerbach, S. M.; Fan, W.; Monson, P. A. Modeling the assembly of nanoporous silica materials. Int. Rev. Phys. Chem. 34 (2015) 35- 70.

[17] Siperstein, F. R.; Gubbins, K. E. Synthesis and Characterization of Templated Mesoporous Materials Using Molecular Simulation. Molec. Simul. 27 (2001) 339-352.

[18] Blin, J. L.; Impéror-Clerc, M. Mechanism of self-assembly in the synthesis of silica mesoporous materials: in situ studies by X-ray and neutron scattering. Chem. Soc. Rev. 42 (2013) 4071-4082.

[19] Hoffmann, F.; Cornelius, M.; Morell, J.; Fröba, M. Silica-Based Mesoporous OrganicInorganic Hybrid Materials. Angew. Chem. Int. Ed. 45 (2006) 3216-3251.

[20] Iler, R.K. Chemistry of Silica. (1979) Wiley-Interscience, New York.

[21] Chen, C. Y.; Burkett, S. L.; Li, H. X.; Davis, M. E. Studies on mesoporous materials II. Synthesis mechanism of MCM-41. Microporous Mater. 2 (1993) 27-34.

[22] Monnier, A.; Schüth, F.; Huo, Q.; Kumar, D.; Margolese, D.; Maxwell, R. S.; Stucky, G. D.; Krishnamurty, M.; Petroff, P.; Firouzi, A.; Janicke, M.; Chmelka, B. F. Cooperative formation of inorganic-organic interfaces in the synthesis of silicate mesostructures. Science 261 (1993) 1299-1303.

[23] Firouzi, A.; Atef, F.; Oertli, A. G.; Stucky, G. D.; Chmelka, B. F. Alkaline Lyotropic Silicate-Surfactant Liquid Crystals. J. Am. Chem. Soc. 119 (1997) 3596-3610. 
[24] Lee, Y. S.; Surjadi, D.; Rathman, J. F. Effects of Aluminate and Silicate on the Structure of Quaternary Ammonium Surfactant Aggregates. Langmuir 12 (1996) 6202-6210.

[25] Albuquerque, A.; Vautier-Giongo, C.; Pastore, H. E. Physical chemistry of nanostructured molecular sieves by the study of phase diagrams: the case of the cetyltrimethylammonium bromide-tetramethylammonium silicate-water system. J. Colloid Interface Sci. 284 (2005) 687693.

[26] Vautier-Giongo, C.; Pastore, H. E. Micellization of CTAB in the presence of silicate anions and the exchange between bromide and silicate at the micelle surface: A step to understand the formation of mesoporous molecular sieves at extremely low surfactant and silicate concentrations. J. Colloid Interface Sci. 299 (2006) 874-882.

[27] Vartuli, J. C.; Schmitt, K. D.; Kresge, C. T.; Roth, W. J.; Leonowicz, M. E.; McCullen, S. B.; Hellring, S. D.; Beck, J. S.; Schlenker, J. L.; Olson, D. H.; Sheppard, E. W. Effect of Surfactant/Silica Molar Ratios on the Formation of Mesoporous Molecular Sieves: Inorganic Mimicry of Surfactant Liquid-Crystal Phases and Mechanistic Implications. Chem. Mater. 6 (1994) 2317-2326.

[28] Zana, R.; Frasch, J.; Soulard, M.; Lebeau, B.; Patarin, J. Fluorescence Probing Investigations of the Mechanism of Formation of Organized Mesoporous Silica. Langmuir 15 (1999) 2603-2606.

[29] Frasch, J.; Lebeau, B.; Soulard, M.; Patarin, J.; Zana, R. In Situ Investigations on Cetyltrimethylammonium Surfactant/Silicate Systems, Precursors of Organized Mesoporous MCM-41-Type Siliceous Materials. Langmuir 16 (2000) 9049-9057.

[30] Galarneau, A.; Di Renzo, F.; Fajula, F.; Mollo, L.; Fubini, B.; Ottaviani, M. F. Kinetics of Formation of Micelle-Templated Silica Mesophases Monitored by Electron Paramagnetic Resonance. J. Colloid Interface Sci. 201 (1998) 105-117. 
[31] Baute, D.; Frydman, V.; Zimmermann, H.; Kababya, S.; Goldfarb, D. Properties of the silica layer during the formation of MCM-41 studied by EPR of a silica-bound spin probe. J. Phys. Chem. B 109 (2005) 7807-7816.

[32] Regev, O. Nucleation events during the synthesis of mesoporous materials using liquid crystalline templating. Langmuir 12 (1996) 4940-4944.

[33] Sadasivan, S.; Fowler, C. E.; Khushalani, D.; Mann, S. Nucleation of MCM-41 nanoparticles by internal reorganization of disordered and nematic-like silica-surfactant clusters. Angew. Chem. Int. Ed. 41 (2002) 2151-2153.

[34] Beurroies, I.; Ågren, P.; Büchel, G.; Rosenholm, J. B.; Amenitsch, H.; Denoyel, R.; Lindén, M. Detailed in situ XRD and calorimetric study of the formation of silicate/mixed surfactant mesophases under alkaline conditions. Influence of surfactant chain length and synthesis temperature. J. Phys. Chem. B 110 (2006) 16254-16260.

[35] Feuston, B. P.; Higgins, J. B. Model Structures for MCM-41 Materials: A Molecular Dynamics Simulation. J. Phys. Chem. 98 (1994) 4459-4462.

[36] Williams, C. D.; Travis, K. P.; Burton N. A.; Harding, J. H. A new method for the generation of realistic atomistic models of siliceous MCM-41. Microporous Mesoporous Mater. 228 (2016) 215-223.

[37] Koh, C. A.; Montanari, T.; Nooney, R. I.; Tahir, S. F.; Westacott, R. E. Experimental and Computer Simulation Studies of the Removal of Carbon Dioxide from Mixtures with Methane Using AlPO4-5 and MCM-41. Langmuir 15 (1999) 6043-6049.

[38] Jing, Y.; Wei, L.; Wang, Y.; Yu, Y. Molecular simulation of MCM-41: Structural properties and adsorption of CO2, N2 and flue gas. Chem. Eng. J. 220 (2013) 264-275.

[39] Siperstein, F. R.; Gubbins, K. E. Phase Separation and Liquid Crystal Self-Assembly in Surfactant-Inorganic-Solvent Systems. Langmuir 19 (2003) 2049-2057.

[40] Larson, R. G.; Scriven, L. E.; Davis, H. T. Monte Carlo simulation of model amphiphileoil-water systems. J. Chem. Phys. 83 (1985) 2411-2420. 
[41] Patti, A.; Mackie, A. D.; Siperstein, F. R. Monte Carlo simulation of self-assembled ordered hybrid materials. Langmuir. 23 (2007) 6771-6780.

[42] Patti, A.; Siperstein, F. R.; Mackie, A. D. Phase behavior of model surfactants in the presence of hybrid particles. J. Phys. Chem. C. 111 (2007) 16035-16044.

[43] Patti, A.; Mackie, A. D.; Zelenak, V.; Siperstein, F. R. One-pot synthesis of amino functionalized mesoporous silica materials: using simulations to understand transitions between different structures. J. Mater. Chem. 19 (2009) 724-732.

[44] Patti, A.; Mackie, A. D.; Siperstein, F. R. Monte Carlo simulations of self-assembling hexagonal and cage-like bifunctional periodic mesoporous materials. J. Mater. Chem. 19 (2009) 7848-7855.

[45] Bhattacharya, S.; Gubbins, K. E. Modeling triblock surfactant-templated mesostructured cellular foams. J. Chem. Phys. 123 (2005) 134907.

[46] Bhattacharya, S.; Coasne, B.; Hung, F. R.; Gubbins, K. E. Modeling micelle-templated mesoporous material SBA-15: atomistic model and gas adsorption studies. Langmuir 25 (2009) $5802-5813$.

[47] Jin, L.; Auerbach, S. M.; Monson, P. A. Simulating the Formation of Surfactant-Templated Mesoporous Silica Materials: A Model with Both Surfactant Self-Assembly and Silica Polymerization. Langmuir 29 (2013) 766-780.

[48] Jorge, M.; Gomes, J. R. B.; Cordeiro, M. N. D. S.; Seaton, N. A. Molecular simulation of silica/surfactant self-assembly in the synthesis of periodic mesoporous silicas. J. Am. Chem. Soc. 129 (2007) 15414-15415.

[49] Jorgensen, W. L.; Gao, J. Monte Carlo Simulations of the Hydration of Ammonium and Carboxylate Ions. J. Phys. Chem. 90 (1986) 2174-2182.

[50] Berendsen, H. J. C.; Grigera, J. R.; Straatsma, T. P. The Missing Term in Effective Pair Potentials. J. Phys. Chem. 91 (1987) 6269-6271. 
[51] Pereira, J. C. G.; Catlow, C. R. A.; Price, G. D. Molecular Dynamics Simulation of Methanolic and Ethanolic Silica-Based Sol-Gel Solutions at Ambient Temperature and Pressure. J. Phys. Chem. A 106 (2002) 130-148.

[52] Gomes, J. R. B.; Cordeiro, M. N. D. S.; Jorge, M. Gas-phase molecular structure and energetics of anionic silicates. Geochim. Cosmochim. Acta 72 (2008) 4421-4439.

[53] Jorge, M.; Gomes, J. R. B.; Cordeiro, M. N. D. S.; Seaton, N. A. Molecular Dynamics Simulation of the Early Stages of the Synthesis of Periodic Mesoporous Silica. J. Phys. Chem. B 113 (2009) 708-718.

[54] Knight, C. T. G. Are zeolite secondary building units really red herrings? Zeolites 10 (1990) 140-144.

[55] Pérez-Sánchez, G.; Gomes, J. R. B.; Jorge, M. Modeling Self-Assembly of Silica/Surfactant Mesostructures in the Templated Synthesis of Nanoporous Solids. Langmuir 29 (2013) $2387-2396$.

[56] Marrink, S. J.; Risselada, H. J.; Yefimov, S.; Tieleman, D. P.; de Vries, A. H. The MARTINI Force Field: Coarse Grained Model for Biomolecular Simulations. J. Phys. Chem. B $2007,111,7812-7824$.

[57] Wu, R.; Deng, M.; Kong, B.; Yang, X. Coarse-Grained Molecular Dynamics Simulation of Ammonium Surfactant Self-Assemblies: Micelles and Vesicles. J. Phys. Chem. B 2009, 113, $15010-15016$.

[58] Jorge, M. Molecular dynamics simulation of self-assembly of $n$-decyltrimethylammonium bromide micelles. Langmuir 24 (2008) 5714-5725.

[59] Chien, S.-C.; Pérez-Sánchez, G.; Gomes, J. R. B.; Cordeiro, M. N. D. S.; Jorge, M.; Auerbach, S. M.; Monson, P. A. Molecular Simulations of the Synthesis of Periodic Mesoporous Silica Phases at High Surfactant Concentrations. J. Phys. Chem. C 121 (2017) $4564-4575$. 
[60] Pérez-Sánchez, G.; Chien, S.-C.; Gomes, J. R. B.; Cordeiro, M. N. D. S.; Auerbach, S. M.; Monson, P. A.; Jorge, M. Multiscale Model for the Templated Synthesis of Mesoporous Silica: The Essential Role of Silica Oligomers. Chem. Mater. 28 (2016) 2715-2727.

[61] Auerbach, S. M.; Ford, M. H.; Monson, P. A. New Insights into Zeolite Formation from Molecular Modeling. Curr. Opin. Colloid Inter. Sci. 10 (2005) 220-225.

[62] Feuston, B. P.; Garofalini, S. H. Empirical three-body potential for vitreous silica. J. Chem. Phys. 89 (1988) 5818-5825.

[63] Rao, N. Z.; Gelb, L. D. Molecular Dynamics Simulations of the Polymerization of Aqueous Silicic Acid and Analysis of the Effects of Concentration on Silica Polymorph Distributions, Growth Mechanisms, and Reaction Kinetics. J. Phys. Chem. B 108 (2004) 12418-12428.

[64] Jing, Z.; Xin, L.; Sun, H. Replica exchange reactive molecular dynamics simulations of initial reactions in zeolite synthesis. Phys. Chem. Chem. Phys. 17 (2015) 25421-25428.

[65] Wu, M. G.; Deem, M. W. Monte Carlo Study of the Nucleation Process during Zeolite Synthesis. J. Chem. Phys. 116 (2002) 2125-2137.

[66] Zhang, X. Q.; Trinh, T. T.; van Santen, R. A.; Jansen, A. P. J. Structure-Directing Role of Counterions in the Initial Stage of Zeolite Synthesis. J. Phys. Chem. C 115 (2011) 9561-9567.

[67] Zhang, X. Q.; van Santen, R. A.; Jansen, A. P. J. Kinetic Monte Carlo modeling of silicate oligomerization and early gelation. Phys. Chem. Chem. Phys. 14 (2012) 11969-11973.

[68] McIntosh, G. J. Theoretical investigations into the nucleation of silica growth in basic solution part II - derivation and benchmarking of a first principles kinetic model of solution chemistry. Phys. Chem. Chem. Phys. 15 (2013) 17496-17509.

[69] Tu, Y.; Tersoff, J. Structure and Energetics of the $\mathrm{Si}_{-S i O_{2}}$ Interface. Phys. Rev. Lett. 84 (2000) 4393-4396.

[70] Burlakov, V. M.; Briggs, G. A. D.; Sutton, A. P.; Tsukahara, Y. Monte Carlo Simulation of Growth of Porous $\mathrm{SiO}_{x}$ by Vapor Deposition. Phys. Rev. Lett. 86 (2001) 3052-3055. 
[71] Malani, A.; Auerbach, S. M.; Monson, P. A. Probing the Mechanism of Silica Polymerization at Ambient Temperatures using Monte Carlo Simulations. J. Phys. Chem. Lett. 1 (2010) 3219-3224.

[72] Malani, A.; Auerbach, S. M.; Monson, P. A. Monte Carlo Simulations of Silica Polymerization and Network Formation. J. Phys. Chem. C. 115 (2011) 15988-16000.

[73] Schumacher, C.; Gonzalez, J.; Wright, P. A.; Seaton, N. A. Generation of Atomistic Models of Periodic Mesoporous Silica by Kinetic Monte Carlo Simulation of the Synthesis of the Material. J. Phys. Chem. B 110 (2006) 319-333.

[74] Ferreiro-Rangel, C. A.; Lozinska, M. M.; Wright, P. A.; Seaton, N. A.; Düren, T. Kinetic Monte Carlo Simulation of the Synthesis of Periodic Mesoporous Silicas SBA-2 and STAC-1: Generation of Realistic Atomistic Models. J. Phys. Chem. C, 116 (2012) 20966-20974.

[75] Huo, Q. S.; Leon, R.; Petroff, P. M.; Stucky, G. D. Mesostructure design with gemini surfactants: supercage formation in a three-dimensional hexagonal array. Science 268 (1995) 1324-1327.

[76] Zhou, W. Z.; Hunter, H. M. A.; Wright, P. A.; Ge, Q. F.; Thomas, J. M. Imaging the pore structure and polytypic intergrowths in mesoporous silica. J. Phys. Chem. B 102 (1998) 69336936.

[77] Bagshaw, S. A.; Prouzet, E.; Pinnavaia, T. J. Templating of mesoporous molecular sieves by nonionic polyethylene oxide surfactants. Science 269 (1995) 1242-1244.

[78] Ryoo, R.; Kim, J. M.; Ko, C. H.; Shin, C. H. Disordered Molecular Sieve with Branched Mesoporous Channel Network. J. Phys. Chem. 100 (1996) 17718-17721.

[79] Yu, C.; Yu, Y.; Zhao, D. Highly ordered large caged cubic mesoporous silica structures templated by triblock PEO-PBO-PEO copolymer. Chem. Commun. 0 (2000) 575-576.

[80] Che, S.; Garcia-Bennett, A. E.; Yokoi, T.; Sakamoto, K.; Kunieda, H.; Terasaki, O.; Tatsumi, T. A novel anionic surfactant templating route for synthesizing mesoporous silica with unique structure. Nature Mater. 2 (2003) 801-805. 
[81] Alexandridis, P.; Hatton, T. A. Poly(ethylene oxide)-poly(propylene oxide)-poly(ethylene oxide) block copolymer surfactants in aqueous solutions and at interfaces: thermodynamics, structure, dynamics, and modeling. Colloids Surf. A Physicochem. Eng. Asp. 96 (1995) 1-46.

[82] Impéror-Clerc, M.; Davidson, P.; Davidson, A. Existence of a microporous corona around the mesopores of silica-based SBA-15 materials templated by triblock copolymers. J. Am. Chem. Soc. 122 (2000) 11925-11933.

[83] Zholobenko, V. L.; Khodakov, A. Y.; Impéror-Clerc, M.; Durand, D.; Grillo, I. Initial stages of SBA-15 synthesis: An overview. Adv. Colloid Interface Sci. 142 (2008) 67-74.

[84] Hezaveh, S.; Samanta, S.; Milano, G.; Roccatano, D. Molecular dynamics simulation study of solvent effects on conformation and dynamics of polyethylene oxide and polypropylene oxide chains in water and in common organic solvents. J. Chem. Phys. 136 (2012) 124901.

[85] Chen, H.; Wu, Y.; Tan, Y.; Li, X.; Qian, Y.; Xi, H. MesoDyn and Experimental Approach to the Structural Fabrication and Pore-size Adjustment of SBA-15 Molecular Sieves. Adsorpt. Sci. Technol. 27 (2009) 579-592.

[86] Yuan, S.; Zhang, X.; Chan. K. Effects of shear and charge on the microphase formation of P123 polymer in the SBA-15 synthesis investigated by mesoscale simulations. Langmuir 25 (2009) 2034-2045.

[87] Chen, H.; Wu, Y.; Tan, Y.; Li, X.; Qian, Y.; Xi, H. Mesoscopic simulation of surfactant/silicate self-assembly in the mesophase formation of sba-15 under charge matching interactions. Eur. Polym. J. 48 (2012) 1892-1900.

[88] Magee, J. E.; Siperstein, F. R. Formation of ordered mesoporous materials under slow aggregation conditions. J. Phys. Chem. C 113 (2009) 1680-1685.

[89] Lettow, J. S.; Han, Y. J.; Schmidt-Winkel, P.; Yang, P.; Zhao, D.; Stucky, G. D.; Ying, J. Y. Hexagonal to Mesocellular Foam Phase Transition in Polymer-Templated Mesoporous Silicas. Langmuir 16 (2000) 8291-8295.

[90] Altevogt, P.; Evers, O. A.; Fraaije, J. G.; Maurits, N. M.; van Vlimmeren, B. A. The 
MesoDyn project: Software for mesoscale chemical engineering. J. Molec. Struct.: THEOCHEM 463 (1999) 139-143.

[91] Sumper, M. A Phase Separation Model for the Nanopatterning of Diatom Biosilica. Science 295 (2002) 2430-2433.

[92] Gröger, C.; Lutz, K.; Brunner, E. Biomolecular self-assembly and its relevance in silica biomineralization. Cell Biochem. Biophys. 50 (2008) 23-39.

[93] Tanev, P. T.; Pinnavaia, T. J. Mesoporous Silica Molecular Sieves Prepared by Ionic and Neutral Surfactant Templating: A Comparison of Physical Properties. Chem. Mater. 8 (1996) $2068-2079$.

[94] Centi, A.; Jorge, M. Molecular Simulation Study of the Early Stages of Formation of Bioinspired Mesoporous Silica Materials. Langmuir 32 (2016) 7228-7240.

[95] Centi, A. Computational Modelling and Design of Bioinspired Silica Materials. PhD Thesis, University of Strathclyde, 2017.

[96] Malliaris, A.; Le Moigne, J.; Sturm, J.; Zana, R. Temperature dependence of the micelle aggregation number and rate of intramicellar excimer formation in aqueous surfactant solutions. J. Phys. Chem 89 (1985) 2709-2713.

[97] Jorgensen, W. L.; Maxwell, D. S.; Tirado-Rives, J. Development and Testing of the OPLS All-Atom Force Field on Conformational Energetics and Properties of Organic Liquids. J. Am. Chem. Soc. 118 (1996) 11225-11236.

[98] Zhang, W.; Pauly, T. R.; Pinnavaia, T. J. Tailoring the Framework and Textural Mesopores of HMS Molecular Sieves through an Electrically Neutral $\left(S^{\circ} I^{\circ}\right)$ Assembly Pathway. Chem. Mater. 9 (1997) 2491-2498.

[99] Hoffmann, F.; Cornelius, M.; Morell, J.; Fröba, M. Silica-based mesoporous organicinorganic hybrid materials. Angew. Chem. Int. Ed. 45 (2006) 3216-3251. 
[100] Pal, N.; Bhaumik, A. Soft templating strategies for the synthesis of mesoporous materials: inorganic, organic-inorganic hybrid and purely organic solids. Adv Colloid Interface Sci. 189190 (2013) 21-41.

[101] Croissant, J. G.; Cattoën, X.; Man, M. W. C.; Durand, J. O.; Khashab, N. M. Syntheses and applications of periodic mesoporous organosilica nanoparticles. Nanoscale, 7 (2015) 20318-20334.

[102] Hatton, B.; Landskron, K.; Whitnall, W.; Perovic, D.; Ozin, G. A. Past, Present, and Future of Periodic Mesoporous Organosilicas - The PMOs. Acc. Chem. Res., 38 (2005) 305312.

[103] Mizoshita, N.; Tani, T.; Inagaki, S. Syntheses, properties and applications of periodic mesoporous organosilicas prepared from bridged organosilane precursors. Chem. Soc. Rev., 40 (2011) 789-800.

[104] Van Der Voort, P.; Esquivel, D.; De Canck, E.; Goethals, F.; Van Driessche, I.; RomeroSalguero, F. J. Periodic Mesoporous Organosilicas: from simple to complex bridges; a comprehensive overview of functions, morphologies and applications. Chem. Soc. Rev., 42 (2013) 3913-3955.

[105] Inagaki S.; Guan S.; Ohsuna T.; Terasaki O. An ordered mesoporous organosilica hybrid material with a crystal-like wall structure. Nature 416 (2002) 304-307.

[106] Futamura, R.; Jorge, M.; Gomes, J. R. B. Structures and energetics of organosilanes in the gaseous phase: a computational study. Theor. Chem. Acc. 132 (2013) 1323-1327.

[107] Futamura, R.; Jorge, M.; Gomes, J. R. B. Role of the organic linker in the early stages of the templated synthesis of PMOs. Phys. Chem. Chem. Phys.,15 (2013) 6166-6169.

[108] Attard, G. S.; Glyde, J. C.; Göltner, C. G. Liquid-crystalline phases as templates for the synthesis of mesoporous silica. Nature 378 (1995) 366-368. 
[109] Taddese, T.; Carbone, P. Effect of Chain Length on the Partition Properties of Poly(ethylene oxide): Comparison between MARTINI Coarse-Grained and Atomistic Models. J. Phys. Chem. B 121 (2017) 1601-1609.

[110] Manning, J. R. H.; Yip, T. W. S.; Centi, A.; Jorge, M.; Patwardhan, S. V. An EcoFriendly, Tunable and Scalable Method for Producing Porous Functional Nanomaterials Designed Using Molecular Interactions. ChemSusChem 10 (2017) 1683-1691.

[111] Pérez-Sánchez, G.; Jorge, M.; Gomes, J. R. B., manuscript in preparation. 